\title{
Effect of deceleration on jet instability
}

\author{
By VLADIMIR SHTERN AND FAZLE HUSSAIN \\ Department of Mechanical Engineering, University of Houston, Houston, TX 77204-4006, USA
}

(Received 14 December 2001 and in revised form 18 November 2002)

A non-parallel analysis of time-oscillatory instability of conical jets reveals important features not found in prior studies. Flow deceleration significantly enhances the shearlayer instability for both swirl-free and swirling jets. In swirl-free jets, flow deceleration causes the axisymmetric instability (absent in the parallel approximation). The critical Reynolds number $R e_{a}$ for this instability is an order of magnitude smaller than the critical $R e_{a}$ predicted before for the helical instability (where $R e_{a}=r v_{a} / v, r$ is the distance from the jet source, $v_{a}$ is the jet maximum velocity at a given $r$, and $v$ is the viscosity). Swirl, intensifying the divergence of streamlines, induces an additional, divergent instability (which occurs even in shear-free flows). For the swirl Reynolds number $R e_{s}$ (circulation to viscosity ratio) exceeding 3, the critical $R e_{a}$ for the single-helix counter-rotating mode becomes smaller than those for axisymmetric and multi-helix modes. Since the critical $R e_{s}$ is less than 10 for the near-axis jets, the boundary-layer approximation (used before) is invalid, as is Long's Type II boundary-layer solution (whose stability has been extensively studied). Thus, the nonparallel character of jets strongly affects their stability. Our results, obtained in a far-field approximation allowing reduction of the linear stability problem to ordinary differential equations, are more valid for short wavelengths.

\section{Introduction}

This paper addresses time-oscillatory instability of strongly non-parallel flows governed by conical similarity solutions of the Navier-Stokes equations. Conical flows include swirl-free round jets (Schlichting 1933; Landau 1944; Squire 1952), swirling jets (Long 1961), and many other flows (e.g. see Shtern \& Hussain 1998, referred to herein as SH98). Prior stability studies of these flows used quasi-parallel and boundary-layer approximations. We show here that critical Reynolds numbers are an order of magnitude smaller than those estimated using quasi-parallel and boundary-layer approximations; both these approximations thus appear invalid.

The steady-state non-parallel analysis (SH98) explained the divergent instability (Goldshtik, Hussain \& Shtern 1991), swirl generation (Shtern \& Barrero 1995), and hysteretic transitions (Shtern \& Hussain 1996), but this analysis did not address the time-oscillatory disturbances which typically are the most dangerous for the swirl-free and swirling jets. To overcome this limitation, we extend here the SH98 approach to generic disturbances by using a far-field approximation. Such approaches are reasonable for stability studies of conical flows which are themselves far-field approximations of practical jets.

Separation of variables applied asymptotically far downstream (i.e. a far-field approximation) seems to have been introduced by Libby \& Fox (1963) who studied the spatial stability of the Blasius boundary layer. Govindarajan \& Narasimha (1995) used similarity variables for the stability study of the Falkner-Skan flows and thus 
took into account weakly nonparallel effects. Tam (1996) applied a similar idea for the spatio-temporal development of disturbances in the plane jet. McAlpine \& Drazin (1998) used the asymptotic separation of variables for spatial stability studies of the Jeffery-Hamel flow in a planar diffuser.

We apply this asymptotic approach to axisymmetric conical flows. The approach is limited to disturbances of wavelengths that are small compared with the distance from the jet origin (this limitation, however, is even more severe in the parallel-flow approximation). An important advantage of the approach is that it involves neither the quasi-parallel nor the boundary-layer approximations of the base flow, while prior stability studies of swirl-free and swirling jets (discussed below) applied one or both of these approximations.

Batchelor \& Gill (1962) studied the stability of swirl-free round jets by considering a top-hat velocity profile close to the nozzle and the Schlichting (1933) solution far from the nozzle. Their inviscid parallel-flow theory revealed no axisymmetric instability of the Schlichting jet and found that only the $m= \pm 1$ helical disturbances can grow; $m$ is the azimuthal wavenumber. Further inviscid and viscous analyses by Kambe (1969), Mollendorf \& Gebhart (1973), Lessen \& Singh (1973), and Morris (1976) also failed to find growing axisymmetric modes. In contrast, we show here that the axisymmetric $(m=0)$ instability does indeed occur and at rather small Reynolds number $R e_{a}$. The $m= \pm 1$ disturbances also grow, but at larger $R e_{a}$ than for the $m=0$ mode. The critical $R e_{a}$ for the $m= \pm 1$ instability estimated using quasi-parallel approximations is nearly twice the value we find by the non-parallel approach.

Stability of swirling jets also has been studied extensively using parallel-flow approximations. One motivation is to explain the vortex breakdown phenomenon. Cores of leading-edge and trailing aircraft vortices, of flows in vortex devices, and of tornadoes (all these cores are swirling jets) can abruptly expand into bubblelike recirculatory zones or into helical or multi-helix patterns-examples of vortex breakdown. The vortex-breakdown mechanism remains an open question despite much work since its discovery (see $\S 4.2$ for a more detailed discussion); one view is that vortex breakdown appears via instability. The fact that tornadoes and delta-wing vortices can be modelled as conical swirling jets has stimulated stability studies of Long's (1961) solution.

Using a boundary-layer approximation for the core of a near-axis swirling flow, Long (1961) found two solution branches (I and II) when the scaled flow force $M>$ $M_{f}=3.74$ and no solution for $M<M_{f}$ (solutions I and II are schematically shown by branches aI and aII respectively in figure 1a). Using a parallel-flow approximation, Foster \& Duck (1982) studied the inviscid stability of solutions I and II near the fold (point $F$ separating aI and aII in figure $1 a$ ); i.e. for $M$ close to $M_{f}$. Foster \& Smith (1989), for solution II, and Ardalan, Draper \& Foster (1995), for solution I, extended the stability study for large $M$. They found that both solutions I and II are unstable to helical disturbances. Foster \& Jacqmin (1992) evaluated weakly nonparallel effects on the inviscid stability characteristics. Khorrami \& Trivedi (1994) corrected some results of Foster \& Duck (1982) and studied weakly viscous effects on flow stability. Using a similar technique, Fernandez-Feria (1996) found the instability of solution II to axisymmetric disturbances. Next, Fernandez-Feria (1999) studied weakly non-parallel spatial instability and found growing disturbances propagating upstream (for solution II).

Whereas these studies of Long's vortex were made in the boundary-layer approximation, Drazin, Banks \& Zaturska (1995) formulated the spatial stability problem using the full Navier-Stokes equations. Shtern \& Hussain (1996) addressed swirling 
(a)
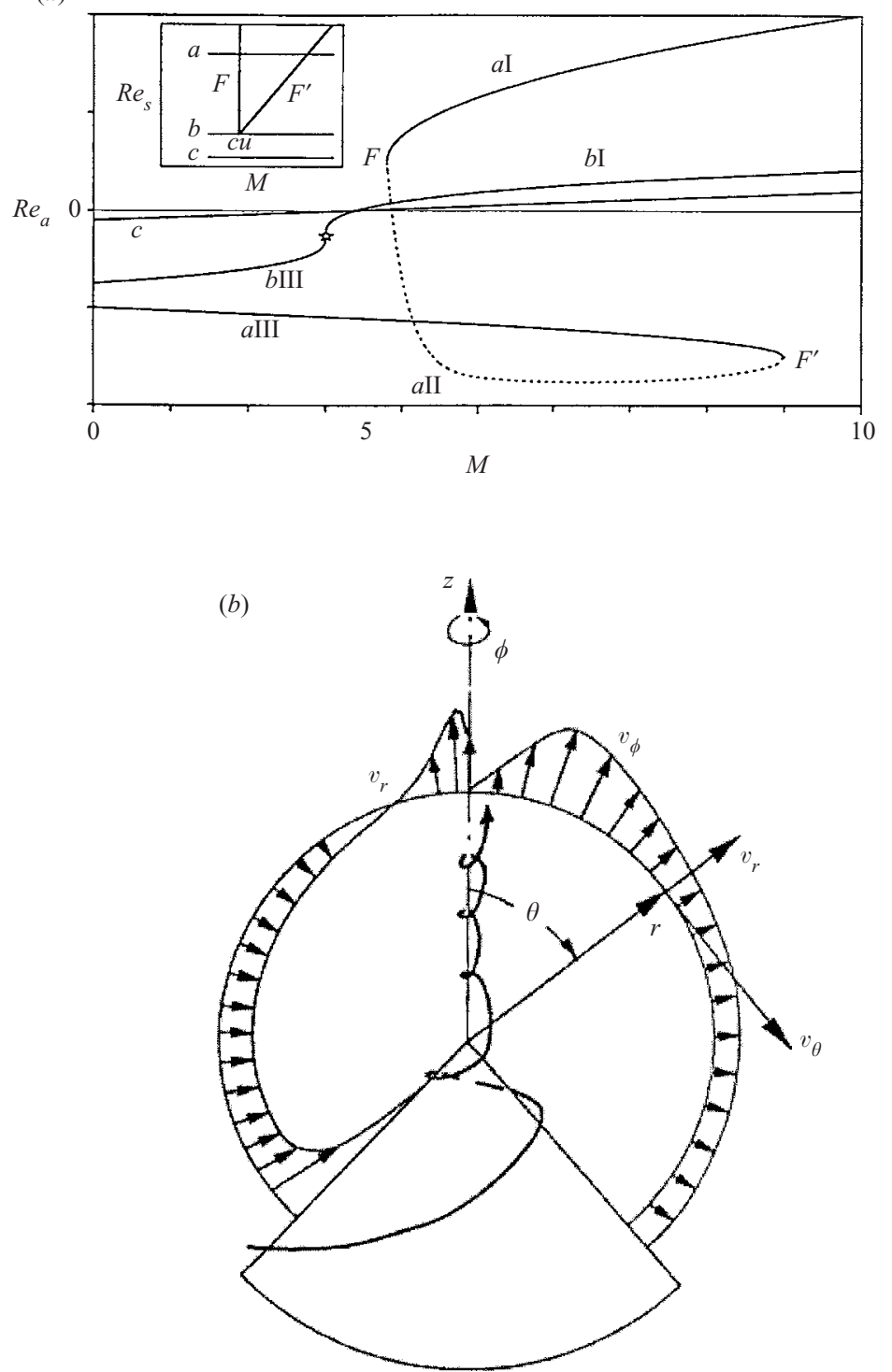

FiguRE 1. (a) Schematic illustrating the disappearance of Long's solution II. Curves $a, b$ and $c$ show the dependence of axial velocity $R e_{a}$ on flow force $M$ at circulation $R e_{s}>R e_{s c u}$, $R e_{s}=R e_{s c u}$, and $R e_{s}<R e_{s c u}$, respectively. $R e_{s c u}$ is the value of $R e_{s}$ at cusp cu (see inset). Curve $a$ consists of branches $a \mathrm{I}, a \mathrm{II}$ and $a \mathrm{III}$; $a \mathrm{II}$ depicts Long's solution II. As decreasing $R e_{s}$ passes $R e_{s c u}, a \mathrm{II}$ degenerates into a point (star symbol on curve $b$ ) and then disappears. (b) Diagram of a base swirling flow outside a cone with coordinates $\{r, \theta, \phi\}$.

flows outside a cone or a half-line vortex. They showed that there are three solutions forming a hysteresis loop (branches aI, aII and aIII in figure 1a), with Long's solution II representing the intermediate branch. This hysteresis occurs only when swirl Reynolds number $R e_{s}$ (circulation to viscosity ratio) is large. As $R e_{s}$ decreases below a cusp value $R e_{s c u}$, the solution becomes fold-free (curve $b$ for $R e_{s}=R e_{s c u}$ and curve $c$ for $R e_{s}<R e_{s c u}$ in figure 1(a); the inset shows the arrangement of solutions 
$a, b$ and $c$ with respect to the cusp, $c u)$; i.e. the hysteresis, hence Long's solution II, disappears. Shtern \& Drazin (2000) studied the spatial stability of the flow induced by a half-line vortex to time-monotonic disturbances. They showed that Long's solution II is unstable to axisymmetric disturbances for $R e_{s}>R e_{s c u}=11.5$, i.e. for all $R e_{s}$ where this solution exists. They also found that Long's solution I is unstable to the $m= \pm 2$ modes when $R e_{s}$ exceeds a critical value dependent on $M$.

Here, we extend this analysis to time-oscillating disturbances (using the full NavierStokes equations). We will show that both the axisymmetric and the $m=-1$ helical instabilities occur for $R e_{s}<R e_{s c u}$. For such small $R e_{s}$, the flow has a unique steady state (i.e. Long's solution II does not exist) and the boundary-layer approach is invalid. That is, flow non-parallelism strongly affects the instability of both swirling and swirl-free jets.

Following the problem formulation in $\S 2$, we study stability of swirl-free $(\S 3)$ and swirling $(\S 4)$ jets and discuss the physical mechanisms of their instabilities $(\S 5)$.

\section{Formulation of the stability problem}

\subsection{Transformation of governing equations}

The stability theory of parallel flows exploits the fact that a base flow depends on only one coordinate (Drazin \& Reid 1981). This permits the normal mode representation of disturbances with respect to other coordinates and time, and thus reduction of the linear stability problem to a system of ordinary differential equations. Axisymmetric conical flows have a feature similar to that of parallel flows: the product, $r \boldsymbol{v}$, depends only on $\theta ; \boldsymbol{v}$ is the velocity vector, $\{r, \theta, \phi\}$ are spherical coordinates, $r$ is the distance from the origin, $\theta$ is the polar angle, and $\phi$ is the azimuthal angle about the axis of symmetry $z$ (figure $1 b$ ). Exploiting this feature, we pursue stability by introducing new dependent variables,

$$
\left.\begin{array}{l}
u(x, \phi, \xi, \tau)=v_{r} r / \nu, \quad v(x, \phi, \xi, \tau)=v_{\theta} r \sin \theta / \nu, \quad \Gamma(x, \phi, \xi, \tau)=v_{\phi} r \sin \theta / \nu, \\
p(x, \phi, \xi, \tau)=\left(\mathscr{P}-\mathscr{P}_{\infty}\right) r^{2} /\left(\rho v^{2}\right),
\end{array}\right\}
$$

where dimensionless functions $u, v, \Gamma$, and $p$ correspond to the velocity components $\left\{v_{r}, v_{\theta}, v_{\phi}\right\}$ and the pressure $\mathscr{P}$, respectively; $\mathscr{P}_{\infty}$ is a constant corresponding to a given pressure at $r \rightarrow \infty, \rho$ is the (constant) density, and $v$ is the kinematic viscosity. New independent variables are

$$
\xi=\ln \left(r / r_{0}\right), \quad x=\cos \theta, \quad \tau=v t / r^{2}
$$

where a length scale $r_{0}$ makes the argument of the logarithm dimensionless. The azimuthal angle $\phi$ is not transformed.

Substitution of (1) and (2) into the Navier-Stokes equations in spherical coordinates (e.g. see Landau \& Lifshitz 1987) yields the system,

$$
\begin{gathered}
u+u_{\xi}-v_{x}+\Gamma_{\phi} /\left(1-x^{2}\right)=2 \tau u_{\tau}, \\
u_{\tau}+u u_{\xi}-u^{2}-v u_{x}+\left(\Gamma u_{\phi}-v^{2}-\Gamma^{2}\right) /\left(1-x^{2}\right) \\
=2 p-p_{\xi}+u_{\xi \xi}+u_{\xi}+\left(1-x^{2}\right) u_{x x}-2 x u_{x}+u_{\phi \phi} /\left(1-x^{2}\right) \\
-2 \tau\left[(1-u) u_{\tau}+2 u_{\xi \tau}-p_{\tau}\right]+4 \tau^{2} u_{\tau \tau},
\end{gathered}
$$




$$
\begin{aligned}
\Gamma_{\tau}+ & u \Gamma_{\xi}-v \Gamma_{x}+\Gamma \Gamma_{\phi} /\left(1-x^{2}\right) \\
= & -p_{\phi}+\Gamma_{\xi \xi}-\Gamma_{\xi}+\left(1-x^{2}\right) \Gamma_{x x}+\left(2 x v_{\phi}+\Gamma_{\phi \phi}\right) /\left(1-x^{2}\right) \\
& -2 \tau\left[2 \Gamma_{\xi \tau}-(1+u) \Gamma_{\tau}\right]+4 \tau^{2} \Gamma_{\tau \tau}, \\
v_{\tau}+ & u v_{\xi}-v u_{\xi}-v u+\left[\Gamma v_{\phi}-v \Gamma_{\phi}-x\left(v^{2}+\Gamma^{2}\right)\right] /\left(1-x^{2}\right) \\
= & \left(1-x^{2}\right) p_{x}+v_{\xi \xi}-v_{\xi}-\left(1-x^{2}\right)\left(u_{x}-u_{x \xi}\right)+\Gamma_{x \phi}+v_{\phi \phi} /\left(1-x^{2}\right) \\
& -2 \tau\left[\left(1-x^{2}\right) u_{x \tau}-(1+u) v_{\tau}+v u_{\tau}+2 v_{\xi \tau}\right]+4 \tau^{2} y_{\tau \tau},
\end{aligned}
$$

where the subscripts denote differentiation with respect to the corresponding variables.

The advantage of (3) is that the coefficients of its steady form (with $\partial / \partial \tau=0$ ) depend on only one independent variable, $x=\cos \theta$, as distinct from the coefficients of the Navier-Stokes equations in spherical coordinates, which depend on two coordinates, $r$ and $\theta$. Unfortunately, system (3) involves terms proportional to $\tau$ and $\tau^{2}$ (these terms appears because $r \partial / \partial r=\partial / \partial \xi-2 \tau \partial / \partial \tau$ ) and, therefore, does not permit the normal-mode representation with respect to $\tau$ for infinitesimal disturbances.

We overcome this difficulty by using a far-field approximation. Consider timeperiodic solutions of period $T$, and let $0 \leqslant t \leqslant T$. Then, $0 \leqslant \tau \leqslant \nu T / r^{2}$ and, therefore, $\tau \rightarrow 0$ as $r \rightarrow \infty$. Thus, terms in (3) proportional to $\tau$ and $\tau^{2}$ become negligible in the far-field approximation, and (3) reduces to:

$$
\begin{gathered}
u+u_{\xi}-v_{x}+\Gamma_{\phi} /\left(1-x^{2}\right)=0, \\
u_{\tau}+u u_{\xi}-u^{2}-v u_{x}+\left(\Gamma u_{\phi}-v^{2}-\Gamma^{2}\right) /\left(1-x^{2}\right) \\
=2 p-p_{\xi}+u_{\xi \xi}+u_{\xi}+\left(1-x^{2}\right) u_{x x}-2 x u_{x}+u_{\phi \phi} /\left(1-x^{2}\right), \\
\Gamma_{\tau}+u \Gamma_{\xi}-v \Gamma_{x}+\Gamma \Gamma_{\phi} /\left(1-x^{2}\right) \\
=-p_{\phi}+\Gamma_{\xi \xi}-\Gamma_{\xi}+\left(1-x^{2}\right) \Gamma_{x x}+\left(2 x v_{\phi}+\Gamma_{\phi \phi}\right) /\left(1-x^{2}\right), \\
v_{\tau}+u v_{\xi}-v u_{\xi}-v u+\left[\Gamma v_{\phi}-v \Gamma_{\phi}-x\left(v^{2}+\Gamma^{2}\right)\right] /\left(1-x^{2}\right) \\
=\left(1-x^{2}\right) p_{x}+v_{\xi \xi}-v_{\xi}-\left(1-x^{2}\right)\left(u_{x}-u_{x \xi}\right)+\Gamma_{x \phi}+v_{\phi \phi} /\left(1-x^{2}\right),
\end{gathered}
$$

where the coefficients depend on $x$ only. Since $u, v, \Gamma$ and $p$ for a base conical flow also depend on $x$ only, we can apply the normal-mode representation with respect to all independent variables $\xi, \phi$ and $\tau$ (except $x$ ) and thus reduce the linear stability problem to a system of ordinary differential equations.

The base flow, a conical similarity solution of the Navier-Stokes equations, can serve as an asymptotic approximation (as $r \rightarrow \infty$ ) for a practical flow, e.g. the Schlichting (1933) solution describes a round jet at large distances from the nozzle. Noting that conical flows are far-field approximations, we use the far-field approximation for their disturbances as well.

\subsection{Equations for infinitesimal disturbances}

A normal mode for infinitesimal perturbations of a base flow can be as follows,

$$
\left.\begin{array}{ll}
u=u_{b}(x)+u_{d}(x) E+\text { c.c., } & v=v_{b}(x)+v_{d}(x) E+\text { c.c., } \\
q=q_{b}(x)+q_{d}(x) E+\text { c.c. }, & \Gamma=\Gamma_{b}(x)+\mathrm{i} \Gamma_{d}(x) E+\text { c.c. },
\end{array}\right\}
$$

where $E=\exp (\alpha \xi+\mathrm{i} m \phi-\mathrm{i} \omega \tau)$, c.c. denotes the complex conjugate of the preceding complex term, complex $\alpha=\alpha_{r}+\mathrm{i} \alpha_{i}$ where $\alpha_{r}$ is the growth of the spatial mode with the radial distance and $\alpha_{i}$ is a radial wavenumber, $m$ is an (integral) azimuthal wavenumber, $\omega$ is the dimensionless frequency, and $b$ and $d$ indicate base flow and disturbance, respectively. 
To compare (5) with the parallel-flow representation for normal modes, it is helpful to substitute, $r=r_{0}+s$, where $r_{0}$ is some reference distance from the jet origin and $s$ is limited to the radial wavelength $L(s<L)$. Assuming that $L / r_{0} \ll 1$ and expanding each of $\xi$ and $\tau$ in power series with respect to $s / r_{0}$, we find from (2) that $\xi=\left[1+O\left(s / r_{0}\right)\right] s / r_{0}$ and $\tau=\left[1+O\left(s / r_{0}\right)\right] v t / r_{0}^{2}$. Thus, for $s / r_{0} \ll 1$, $\xi$ and $\tau$ are the dimensionless local coordinate and time, respectively, similar to those used in the quasi-parallel stability studies. For long waves $\left(L / r_{0} \geqslant 1\right)$, this interpretation and the far-field approximation are both invalid, but the parallel-flow approach is even worse because it ignores the base-flow divergence. We conclude that the far-field approximation is valid at least where the parallel approach is valid and, additionally, accounts for the base-flow deceleration. Based on this and the abovementioned expansions, we interpret $\omega$ and $\alpha$ as dimensionless frequency and the radial wavenumber (as in the quasi-parallel stability theories).

The real part $\alpha_{r}$ of exponent $\alpha$ characterizes the spatial stability. As $r$ increases, if $\alpha_{r}<0$, the disturbance decays faster than the base flow; if $\alpha_{r}=0$, the disturbance amplitude and the base flow have the same $r$-dependence (their velocities decay as $\left.r^{-1}\right)$; and if $\left(\alpha_{r}>0\right.$, the ratio of disturbance to the base flow amplitude increases with $r$. Hence, $\alpha_{r}<0, \alpha_{r}=0$ and $\alpha_{r}>0$ correspond to spatial stability, neutral stability and instability of the base flow, respectively.

Substitution of (5) and simple calculations reduce the linearized version of (4) to the following system of ordinary differential equations:

$$
\begin{aligned}
v_{d}^{\prime}= & (1+\alpha) u_{d}-m \Gamma_{d} /\left(1-x^{2}\right) \\
\left(1-x^{2}\right) u_{d}^{\prime \prime}= & \left(2 x-v_{b}\right) u_{d}^{\prime}+\left[(\alpha-2) u_{b}+p_{1}-\alpha-\alpha^{2}-\mathrm{i} \omega\right] u_{d} \\
& -u_{b}^{\prime} v_{d}+(\alpha-2) p_{d}-p_{4} \\
\left(1-x^{2}\right) \Gamma_{d}^{\prime \prime}= & \left(\alpha u_{b}+p_{5}\right) \Gamma_{d}-v_{b} \Gamma_{d}^{\prime}+\left(\mathrm{i} \Gamma_{b}-p_{2}\right) v_{d}-2 m u_{d}+m p_{d} \\
\left(1-x^{2}\right) p_{d}^{\prime}= & \left(1-x^{2}\right)(1-\alpha) u_{d}^{\prime}+\left[p_{5}-(1-\alpha) u_{b}\right] v_{d} \\
& -(1+\alpha) v_{b} u_{d}-x p_{4}+m \Gamma_{d}^{\prime}+p_{3} \Gamma_{d}
\end{aligned}
$$

where

$$
\begin{gathered}
p_{1}=\left(\mathrm{i} m \Gamma_{b}+m^{2}\right) /\left(1-x^{2}\right), \quad p_{2}=2 m x /\left(1-x^{2}\right), \quad p_{3}=m v_{b} /\left(1-x^{2}\right), \\
p_{4}=2\left(v_{b} v_{d}+\mathrm{i} \Gamma_{b} \Gamma_{d}\right) /\left(1-x^{2}\right), \quad p_{5}=\alpha-\alpha^{2}+p_{1}-\mathrm{i} \omega,
\end{gathered}
$$

and the prime denotes differentiation with respect to $x(=\cos \theta)$. We have reordered (4) to a form convenient for numerical integration by putting the terms with the highest derivatives on the left-hand side and all other terms on the right-hand side of (6).

\subsection{Boundary conditions}

We consider a flow outside a cone, $x<x_{c}$, with the cone tip located at the coordinate origin and the cone axis aligned with the axis of symmetry, $z$ (figure $1 b$ ). Therefore, the flow region is $x_{c} \leqslant x \leqslant 1, x_{c} \geqslant-1$. When $x_{c}=-1$, the flow occupies the entire space. Since system (6) is of the sixth order, we need six boundary conditions: three of them must be satisfied at $x=1$ and the other three at $x=x_{c}$. These conditions are listed below.

The axis of symmetry corresponds to the singularity points, $x= \pm 1$, for (6). If the problem formulation prescribes no singularity on the axis, the solution must be regular there. This leads to the conditions on the positive $z$-axis, $x=1$ :

$$
v_{d}=\Gamma_{d}=0 .
$$


Next, $(6 b)$ yields, at $x=1$,

$$
\left.\begin{array}{l}
u_{d}=0 \quad \text { for } m \neq 0, \\
f_{d} \equiv 2 x u_{d}^{\prime}+\left[(\alpha-2) u_{b}-\alpha-\alpha^{2}-\mathrm{i} \omega\right] u_{d}+(\alpha-2) p_{d}=0 \quad \text { for } m=0 .
\end{array}\right\}
$$

If $x_{c}=-1$, and there is no singularity at $x=-1$ (e.g. in the case of a swirl-free jet from a point source of momentum, see Landau \& Lifshitz 1987), conditions (7a) and $(7 b)$ must be satisfied at $x=-1$ as well. In the case of a half-line vortex, where $\Gamma_{b} \neq 0$ and $u_{b}$ has a logarithmic singularity at $x=-1$ (e.g. see Shtern \& Drazin 2000), disturbances must satisfy the conditions,

$$
v_{d}(-1)=\Gamma_{d}(-1)=u_{d}(-1)=0 .
$$

At $x=x_{c} \neq-1$, disturbances satisfy either no-slip or stress-free conditions. The no-slip condition on the cone surface yields

$$
v_{d}=\Gamma_{d}=u_{d}=0 \quad \text { at } x=x_{c} .
$$

The impermeability and stress-free conditions yield (SH98)

$$
v_{d}=u_{d}^{\prime}=\left(1-x^{2}\right) \Gamma_{d}^{\prime}+2 x \Gamma_{d}=0 \quad \text { at } x=x_{c} .
$$

In the case of the Landau jet or the Squire jet, the flow force is given. As the flow is disturbed, disturbances must satisfy the condition that they provide no additional flow force. For steady axisymmetric disturbances, this would require an additional integral condition. However, for disturbances studied here, those are periodic with respect to $t$ and/or with respect to $\phi$, this integral requirement is automatically satisfied.

\subsection{Eigenvalue problem}

The conditions for disturbances, e.g. (7a)-(7c), and equations (6) form a mathematically closed problem which admits the trivial solution, $u_{d}=v_{d}=q_{d}=\Gamma_{d}=0$. To find a non-trivial (eigen) solution for the normal modes, we should seek complex eigenvalues of either $\alpha$ for a given real $\omega$ (spatial stability) or $\omega$ for $\alpha_{r}=0$ (temporal stability). This paper focuses on neutral disturbances, for which the results of the spatial and temporal stability approaches are identical (since $\alpha_{r}=0$ and $\omega$ is real). However, to find neutral characteristics, we use the spatial stability approach because all eigenvalues of $\alpha$ are known for $R e_{a}=R e_{s}=\omega=0$ and any $m$ (SH98). Eventually, by increasing the axial and/or swirl Reynolds numbers, $R e_{a}$ and $R e_{s}$ (which characterize the strength of the base flow), as well as frequency $\omega$, we find $\alpha$ by the Newton shooting procedure using the $\alpha$ value found at previous parameter values for an initial guess. Applying this algorithm for a few spectral branches (that have the largest $\alpha_{r}$ at $R e_{a}=R e_{s}=0$ ) we find what disturbance mode is the most dangerous, i.e. have the smallest critical values of $R e_{a}$ and $R e_{s}$.

In the shooting procedure, we start at an intermediate location, $x=x_{0}$, with some tentative values of $v_{d}, p_{d}, u_{d}, \Gamma_{d}, u_{d}^{\prime}$ and $\Gamma_{d}^{\prime}$, and integrate (6) in both directions: to $x=1$ and to $x=x_{c}$. Then, we adjust the tentative values to satisfy a normalization condition (e.g. $v_{d}\left(x_{0}\right)=1$ ) and the boundary conditions except one (e.g. $v_{d}\left(x_{c}\right)=0$ ) by the Newton shooting procedure (these shooting iterations rapidly converge starting with any tentative values because the problem is linear). Then, we look for an eigenvalue $\alpha$ to satisfy the postponed boundary condition (e.g. $\left.v_{d}\left(x_{c}\right)=0\right)$.

For numerical integration we apply the fourth-order Runge-Kutta algorithm and the Chebyshev grid whose mesh decreases where required (i.e. within boundary layers for large $R e$ ). The total number of grid points was typically 800 . We also use 1600 grid points to validate accuracy. 


\section{Stability of swirl-free jets}

\subsection{Stability of the Landau jet}

First, we consider the stability of a swirl-free jet in an unbounded region driven by a point source of momentum located at the coordinate origin.

\subsubsection{Base flow}

This flow is described by the exact solution of the Navier-Stokes equations (Landau \& Lifshitz 1987):

$$
v_{b}=-2\left(1-x^{2}\right) /\left(1-x+4 / \operatorname{Re}_{a}\right),
$$

where $R e_{a}=u_{b}(1)=r v_{a} / v\left(v_{a}=v_{r}\right.$ at $\left.x=1\right)$ as follows from

$$
u_{b}=v_{b}^{\prime}=\left[16 \operatorname{Re}_{a} x-2 \operatorname{Re}_{a}^{2}(1-x)^{2}\right] /\left[\operatorname{Re}_{a}(1-x)+4\right]^{2},
$$

and thus $R e_{a}$ is the Reynolds number based on the velocity on the positive $z$-axis and the distance from the jet source.

As $R e_{a} \rightarrow \infty$ while $\eta=R e_{a}(1-x)$ remains bounded, $(8 b)$ yields

$$
u_{\text {in }}=16 \operatorname{Re}_{a} /(4+\eta)^{2} \text {, }
$$

which coincides with the Schlichting (1933) solution for a round jet. Subscript in in $(8 c)$ denotes the inner (boundary-layer) solution. Outside the near-axis boundary layer, $(8 a)$ and $(8 b)$ give

$$
v_{\text {out }}=-2(1+x), \quad u_{\text {out }}=-2,
$$

as $R e_{a} \rightarrow \infty$; subscript out denotes the outer solution describing the flow induced by a half-line sink of fluid located along the positive $z$-axis (i.e. the entrainment flow of the jet).

\subsubsection{Stability}

Figure 2 depicts the results for neutral $\left(\alpha_{r}=0\right)$ disturbances. We have not found neutral disturbances for azimuthal wavenumbers $m$ other than $m=0$ and $m= \pm 1$ (the results of $\S 3$ are independent of the sign of $m$ ). In figure 2(a), the solid (dashed) curves represent the dependence of radial wavenumber $\alpha_{i}$ (frequency $\omega$ ) on Reynolds number $\operatorname{Re}_{a}\left(\alpha R\right.$ denotes the product $\left.\alpha_{i} R e_{a}\right)$. We see that the axisymmetric $(m=0)$ neutral disturbances have the critical Reynolds number, wavenumber and frequency smaller than those for the helical mode.

Flow deceleration enhances the disturbance growth (via the mechanism discussed later in this section). This enhancement, being larger for the axisymmetric disturbances than for helical disturbances in swirl-free jets, makes the axisymmetric mode dominant.

In figure $2(b)$ we compare our results for the helical mode (solid curve) with those obtained in the parallel-flow approximation for the Schlichting jet (dashed curve, Morris 1976). For the minimum (critical) Reynolds number our results are: $R e_{a c}=101, \alpha_{i}=1.85$, and $\omega=84$ while Morris's results are: $R e_{a c}=177, \alpha_{i}=2.2$, and $\omega=83$; the largest difference is in $R e_{a c}$ and the least in $\omega$.

To observe the asymptotic trend as $R e_{a} \rightarrow \infty$, we use Morris's parameters (denoted by subscript $M$ ): Reynolds number $R_{M}=\left(8 R e_{a}\right)^{1 / 2}, \alpha_{M}=\alpha_{i}\left(8 / R e_{a}\right)^{1 / 2}$ and $\omega_{M}=$ $\omega\left(8 / R e_{a}^{3}\right)^{1 / 2}$. The curves in figure $2(b)$ differ in two significant features:

(A) In our case, the upper branch has a local maximum $\left(\omega_{M}=1.15\right.$ at $\left.R_{M}=150\right)$ and the lower branch has a local minimum $\left(\omega_{M}=0.037\right.$ at $\left.R_{M}=95\right)$ while the parallel-flow theory gives monotonic variations of $\omega_{M}$ as $R_{M}$ increases. 


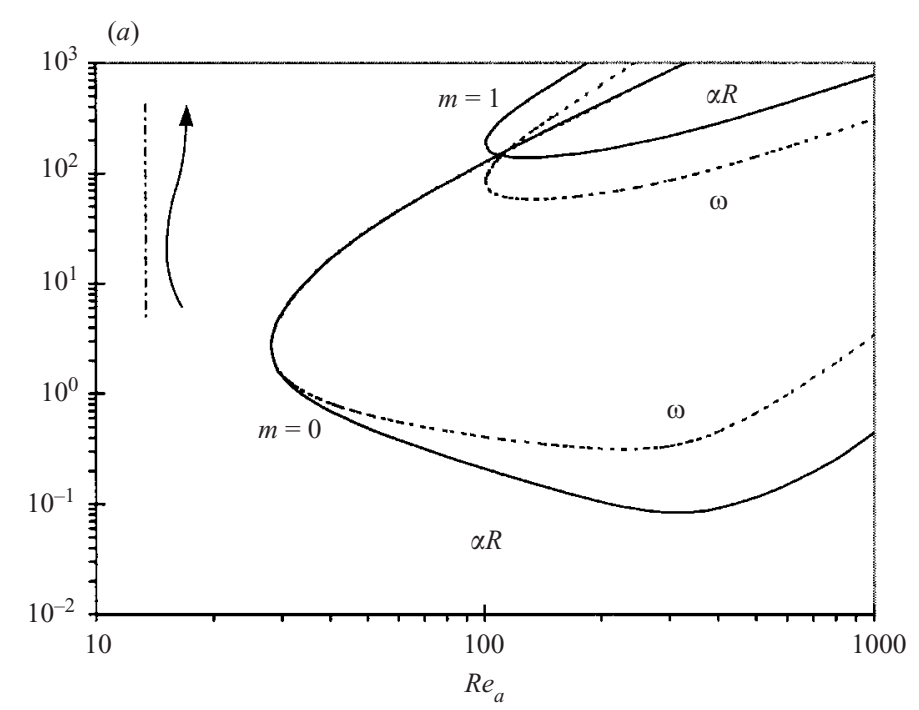

(b)

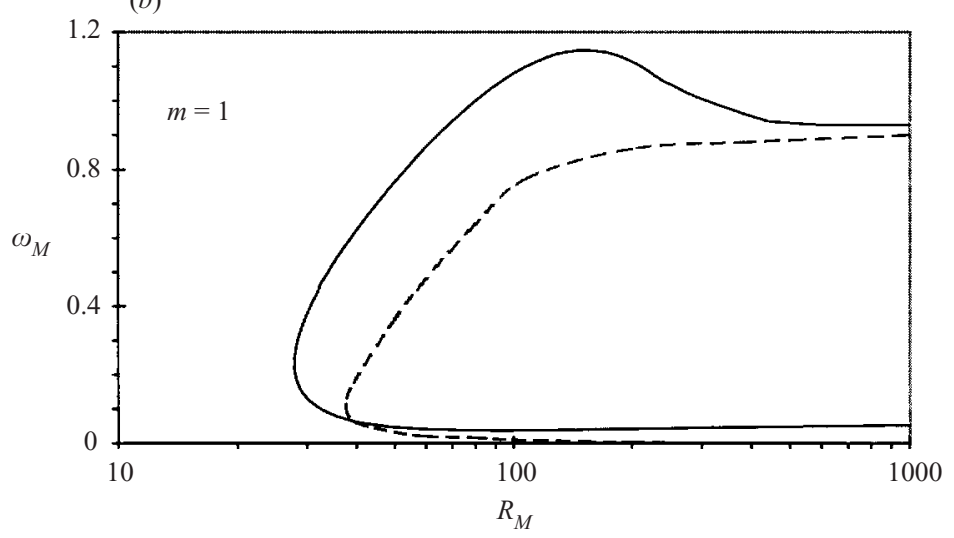

Figure 2. $(a)$ Neutral curves $\left(\alpha_{r}=0\right)$ of axisymmetric $(m=0)$ and helical $(m=1)$ disturbances of the Landau jet (inset), $\omega$ is the frequency, $\alpha R \equiv \alpha_{i} R e_{a}, \alpha_{i}$ is the radial wavenumber, and $R e_{a}$ is the Reynolds number based on the velocity on the axis and the distance $r$ from the jet source. $(b)$ Comparison with the parallel-flow results (---, Morris 1976). The inset in $(a)$ shows a flow schematic: a streamline (solid), the flow direction (arrow), and the symmetry axis (dotted).

(B) Our instability ranges for $\omega_{M}$ and $R_{M}$ (also $\alpha_{M}$ ) are larger than those for the parallel theory.

Two factors can cause this difference: (i) the boundary-layer approximation (Schlichting jet versus Landau jet) and (ii) the parallel-flow approximation. To help evaluate the role of (i), figure $3(a)$ shows the radial velocity $v_{r}$ for both the Landau (solid curve) and Schlichting (dashed curve) solutions as well as kinetic energy $E_{d}=\left|u_{d}\right|^{2}+\left|v_{d}\right|^{2} /\left(1-x^{2}\right)$ of the neutral disturbance (all normalized by their maximum values at a given $r$ ) as functions of polar angle $\theta$ at $R e_{a c}=101$. The disturbance energy peaks near the inflection point, where the base-flow shear reaches its maximum, and $E_{d}$ decreases as the shear decreases. Moreover, the profiles of $E_{d}$ and of the squared base-flow shear (being normalized by their maxima) nearly coincide. This suggests the shear-layer nature of this instability. Since the $v_{r}$ profiles 

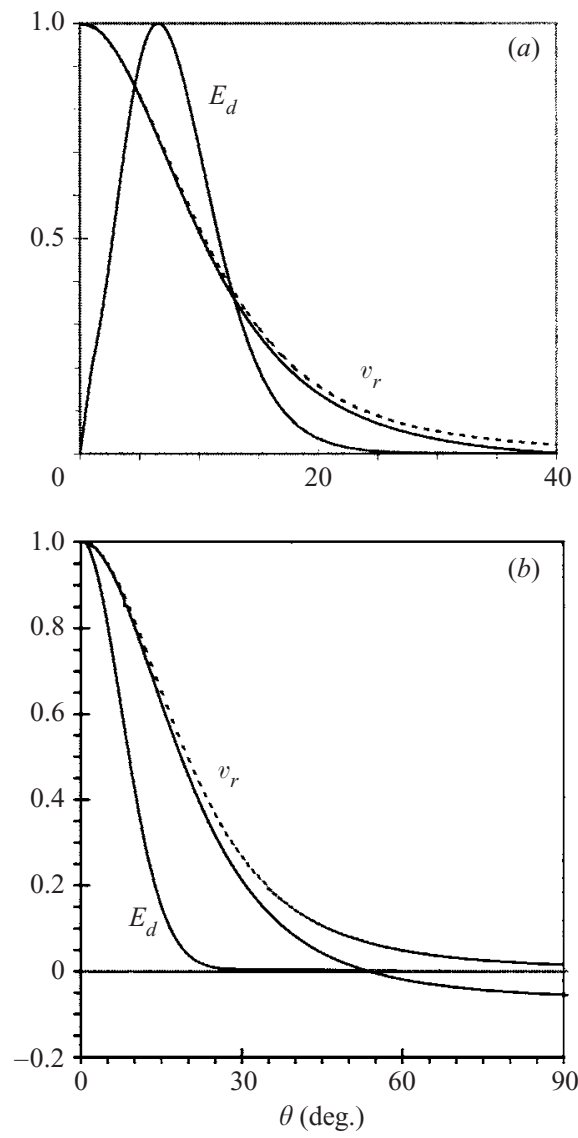

FIGURE 3. Dependence of the radial velocity $v_{r}$ of the free round jet (-, Landau; ---, Schlichting) and the energy $E_{d}$ of $(a)$ neutral helical $\left(R e_{a}=101\right)$ and $(b)$ neutral axisymmetric $\left(R e_{a}=28.1\right)$ disturbances.

for Landau's and Schlichting's solutions are very close within the cone, $\theta \leqslant 25^{\circ}$ (which includes the high-shear region), the role of factor (i) appears negligible here. In contrast, factor (ii) - the base-flow non-parallelism - is crucial, as discussed below.

Flow deceleration effect. To illustrate the effect of flow deceleration, we represent a disturbed streamline by a wavy curve (figure $4 a$ ). Stretching of the streamline by acceleration $\left(\partial v_{b r} / \partial r>0\right)$ decreases the wave amplitude (figure $\left.4 b\right)$; compression of the streamline by deceleration $\left(\partial v_{b r} / \partial r<0\right)$ increases the amplitude (figure $4 c$ ), thus enhancing the instability (see $\S 5$ for more detailed discussion of the deceleration effect).

In swirl-free flows, the base-flow deceleration does not affect the spanwise disturbance velocity. In terms of vorticity, the deceleration - by compressing the flow in the axial direction and stretching it in the normal direction - decreases the axial component $\left(\omega_{r}\right)$ and increases the azimuthal component, $\omega_{\phi}$. This suggests why the effect of the base-flow deceleration is stronger for axisymmetric modes (where only $\omega_{\phi} \neq 0$ ) than for helical modes (where $\omega_{r} \neq 0$ ). (The latter effect is analogous to vortex rings impinging on a plate where the ring radius increase accentuates $\omega_{\phi}$ and hence $v_{r}$.) 
(a)

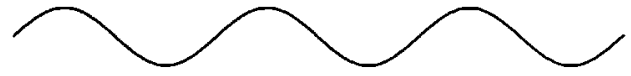

(b)

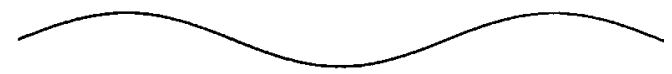

(c)

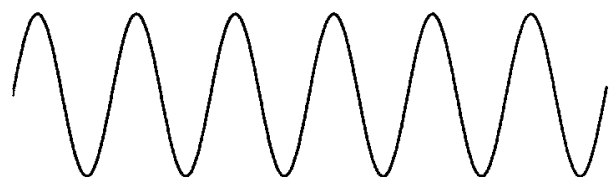

FiguRE 4. Diagram of $(a)$ a parallel-flow disturbance and its $(b)$ suppressed and $(c)$ amplified forms induced by the base flow acceleration (stretching, $b$ ) and deceleration (compression, $c$ ).

Thus, the base-flow deceleration by increasing the disturbance growth expands the instability range for all the parameters $\left(R e_{a}, \alpha_{i}\right.$, and $\left.\omega\right)$; this explains feature $(B)$. Concerning feature $(A)$, the base-flow shear, i.e. the term $r^{-1} v_{d \theta} \partial v_{b r} / \partial \theta$, plays an important role. As $R e_{a}$ increases, the jet becomes thin in the $\theta$-direction, so that the shear, $r^{-1} \partial v_{b r} / \partial \theta$, increases while the deceleration, $\partial v_{b r} / \partial r$, does not. Therefore, the term, $r^{-1} v_{d \theta} \partial v_{b r} / \partial \theta$, dominates the term, $v_{d r} \partial v_{b r} / \partial r$, i.e. the role of the base-flow deceleration (which is the main non-parallel effect) diminishes and the instability range becomes smaller and close to that in the parallel theory; this explains feature $(A)$.

Short waves are less sensitive to the flow non-parallelism than long waves, because the disturbance acceleration $v_{b r} \partial v_{d r} / \partial r$ becomes large for high wavenumbers and also diminishes the role of the base flow acceleration $v_{d r} \partial v_{b r} / \partial r$. For this reason, the convergence of our results to parallel ones, as $R_{M}$ increases, is faster for the upper (than for the lower) branch of the neutral curve (figure $2 b$ ). For example, $\omega_{M}=0.93$ and $\alpha_{M}=1.49$ at $R_{M}=500$ on the upper branch are close to $\omega_{M}=0.91$ and $\alpha_{M}=1.46$ at $R_{M}=\infty$ (according to the parallel theory, Batchelor \& Gill 1962). (This agreement is an additional validation of our numerical procedure (note that the shooting method we use has poor convergence for very large $R_{M}$ ).) Our calculations show that $\omega_{M}$ and $\alpha_{M}$ increase with $R_{M}$ for large $R_{M}$ along the lower branch, e.g. $\omega_{M}=0.052$ and $\alpha_{M}=0.0814$ at $R_{M}=1000$.

Thus, our results approach those from the parallel-flow theory as the Reynolds number increases along the upper branch of the neutral curve; however, they reveal (a) larger instability ranges for $\omega_{M}$ and $\alpha_{M}$ and $(b)$ smaller critical $R_{M}$ than those in the parallel-flow theory; both these effects are due to base-flow deceleration.

For axisymmetric $(m=0)$ disturbances, the difference between our and the parallelflow results is even more significant than for helical modes. Previous studies have revealed no axisymmetric instability ( indeed occurs. Furthermore, it occurs for smaller $R e_{a}$ than helical instability does; i.e. axisymmetric disturbances are the most dangerous. (We have explained why $\omega_{\phi}$ amplification is larger for axisymmetric ring-like vorticity perturbations.)

The fold of the $m=0$ curves in figure 2(a) corresponds to $R e_{a c}=28.1$ where $\alpha_{i}=0.097$ and $\omega=2.71$. Although $R e_{a c}$ for $m=0$ is significantly smaller than $R e_{a c}$ for $m=1$ and therefore the role of viscous diffusion increases, the disturbance energy $E_{d}$ is again localized in a narrow range of $\theta$. This is clear from figure $3(b)$ which also shows that, despite the fact that the $v_{r}$ profiles for Landau's (solid curve) and Schlichting's (dashed curve) differ away from the axis at $R e_{a}=28.1$, the profiles are 

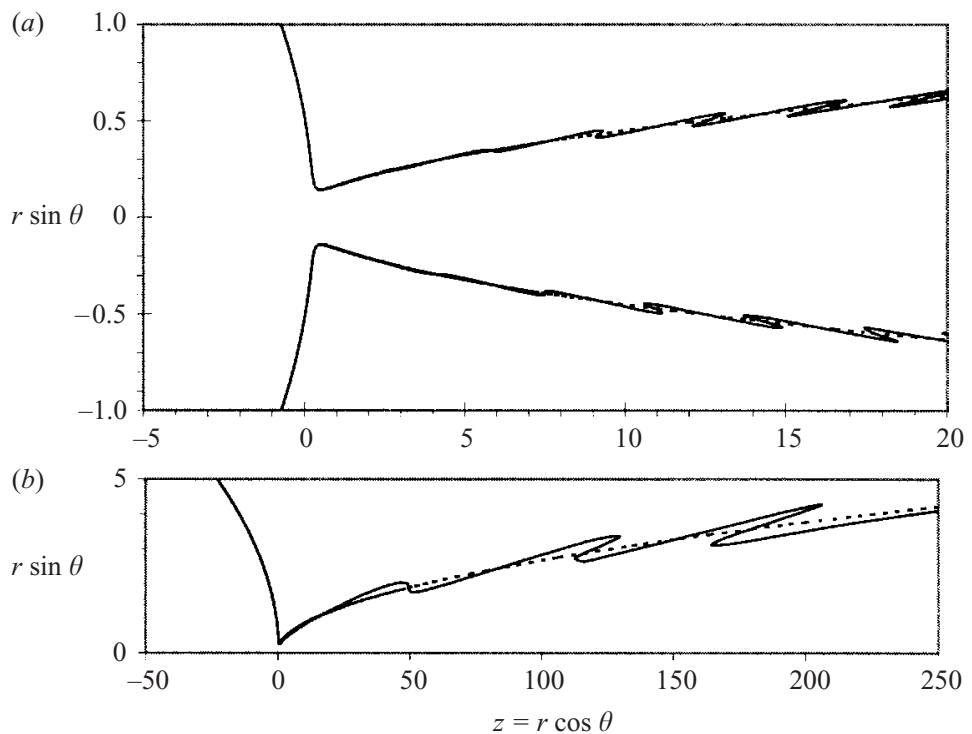

Figure 5. Meridional ( $\phi=$ const) cross-section of ---, undisturbed - , and disturbed stream surfaces of the Landau jet for $(a) m=1$ and $(b) m=0$ modes. Amplitudes of the disturbances are enlarged for better visibility. Note that the wavelength of the axisymmetric mode is about 20 times that of the helical mode.

still close near the axis $\left(\theta<20^{\circ}\right)$ where $E_{d}$ is localized. Therefore, similar to helical modes, the boundary-layer approximation (introducing very small profile change) cannot be the reason for the difference between our and the parallel-flow results concerning the axisymmetric instability.

In contrast to the $\theta$-extent, the streamwise wavelength, $2 \pi / \alpha_{i}$, of the axisymmetric critical disturbances is remarkably larger than that of helical disturbances, as figure 5 illustrates. This is a side effect of the decrease in $R e_{a c}$ (from 101 to 28); viscous dissipation suppresses disturbances of even small wavenumber as $R e_{a}$ decreases and thus $\alpha_{i}$ of growing waves decreases. Figure 5 shows the $\phi=$ constant crosssections of stream surfaces for $(a)$ helical and $(b)$ axisymmetric neutral disturbances at $R e_{a}=101$ and $R e_{a}=28.1$, respectively. The dashed curves depict undisturbed stream surfaces while the solid curves depict disturbed ones; (infinitesimal) disturbances are exaggerated here for better clarity. Streamlines are undisturbed upstream of the jet source $(z<0)$ where the flow accelerates while the oscillations increase downstream proportional to $r$ (although oscillation amplitudes are fixed). Figure 5 also illustrates the difference between our similarity modes (whose spatial size is scaled by a local value of $r$ and, accordingly, increases with increasing $r$ ) and the parallel-flow modes (whose wavelength is invariant downstream).

Another feature is that waves on streamlines in figure 5 have a tendency to overturn as the disturbances travel downstream. This occurs because the wave-propagation velocity $c=\omega / \alpha_{i}$ is $\theta$-independent while the jet velocity $v_{r}$ decreases as $\theta$ increases, so $c / v_{r}$ increases with $\theta$ causing the peaks (maximums of the distance from the axis) to move faster than the valleys relative to the local fluid velocity.

An interesting effect of the non-parallel flow is that $c$ can be larger than $v_{r m}$ (the maximum of $v_{r}$ at a given $r$ ) as figure 6 shows, where $C=c / v_{r m}$. This differs from $C<1$ in the parallel-flow stability theory (Drazin \& Reid 1981, p. 142). (Note that 


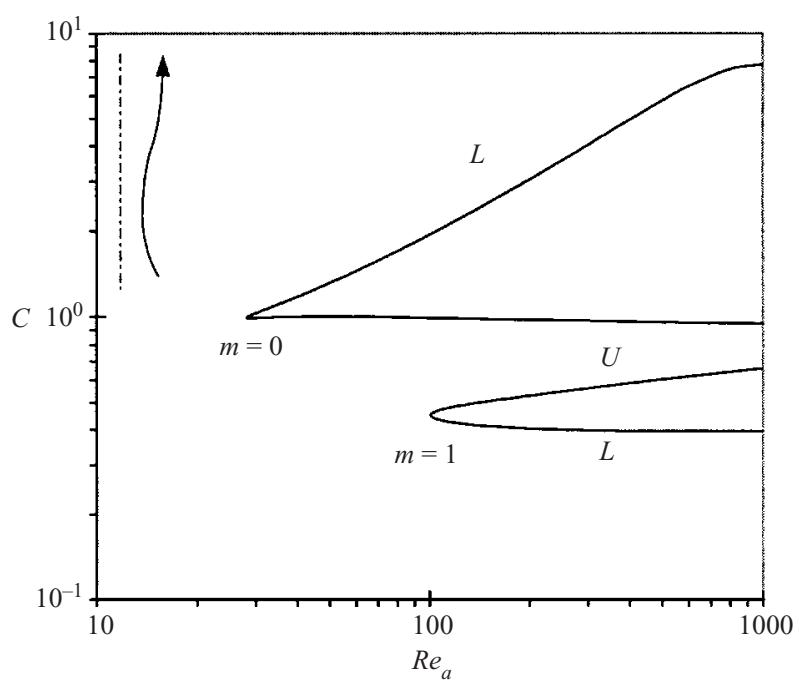

FIGURE 6. Phase speed $C$ of neutral disturbances in the radial direction for a Landau jet (inset); $C$ is scaled by the maximum radial velocity at a given $r$.

$U$ and $L$ are used to denote the upper (large $\omega$ ) and lower (small $\omega$ ) branches of neutral curves; for $m=1, U$ and $L$ notations are the usual, but for the $m=0$ curves in figures 6 and $7(b)$, their relative positions are opposite.) While for helical ( $m=1$ ) disturbances, $C<1$ on both the $U$ and $L$ branches of the neutral curve, $C$ significantly exceeds 1 for the axisymmetric modes on the $L$ branch of the neutral curve in figure 6 , where $\alpha_{i}$ is very small (see figure 2 ); i.e. the wavelength is very large (the upper branch of the $m=0$ neutral curve in figure 6 corresponds to the lower branch in figure 2). A possible reason for $C>1$ is that long waves have more inertia than short waves. As the neutral disturbances propagate from the high- to low-speed flow regions, long waves retain their high momentum gained upstream, whereas short waves, being less inertial, adjust their momentum to the local velocity of the flow.

Note, however, that the far-field approximation is questionable for very long waves because their length can exceed the distance to the jet source, and therefore the nearfield flow region and the nozzle geometry (neglected in the far-field approximation) can influence the stability characteristics by decreasing the speed of wave propagation.

The wavelength of axisymmetric critical disturbances is larger than that of helical disturbances and according to the vortex-ring analogy, the flow non-parallelism effect on the axisymmetric modes is stronger than on helical disturbances. Our calculations have confirmed that in the parallel-flow approximation as well as in weakly nonparallel approximation, no axisymmetric instability occurs. Thus, only the strongly non-parallel approach reveals the axisymmetric instability.

\subsubsection{Comparison with experimental data}

Experimental data on the round jet stability have been obtained in terms of $R e_{D}$, the Reynolds number based on the flow-rate velocity and the nozzle diameter. The relation between $R e_{D}$ and the far-field $R_{M}\left[=\left(3 J / \pi \rho v^{2}\right)^{1 / 2} ; J\right.$ is the flow force] depends on the velocity profile at the nozzle exit: $R e_{D}=R_{M}$ and $R e_{D}=R_{M} 2 / \sqrt{ } 3$ for the parabolic (laminar) and top-hat (turbulent) distributions, respectively (Morris 1976). Since the coefficients are rather close and the parabolic distribution is more suitable for the laminar jets studied here, we take for comparison $R e_{D}=R_{M}=\left(8 R e_{a}\right)^{1 / 2}$ (the 

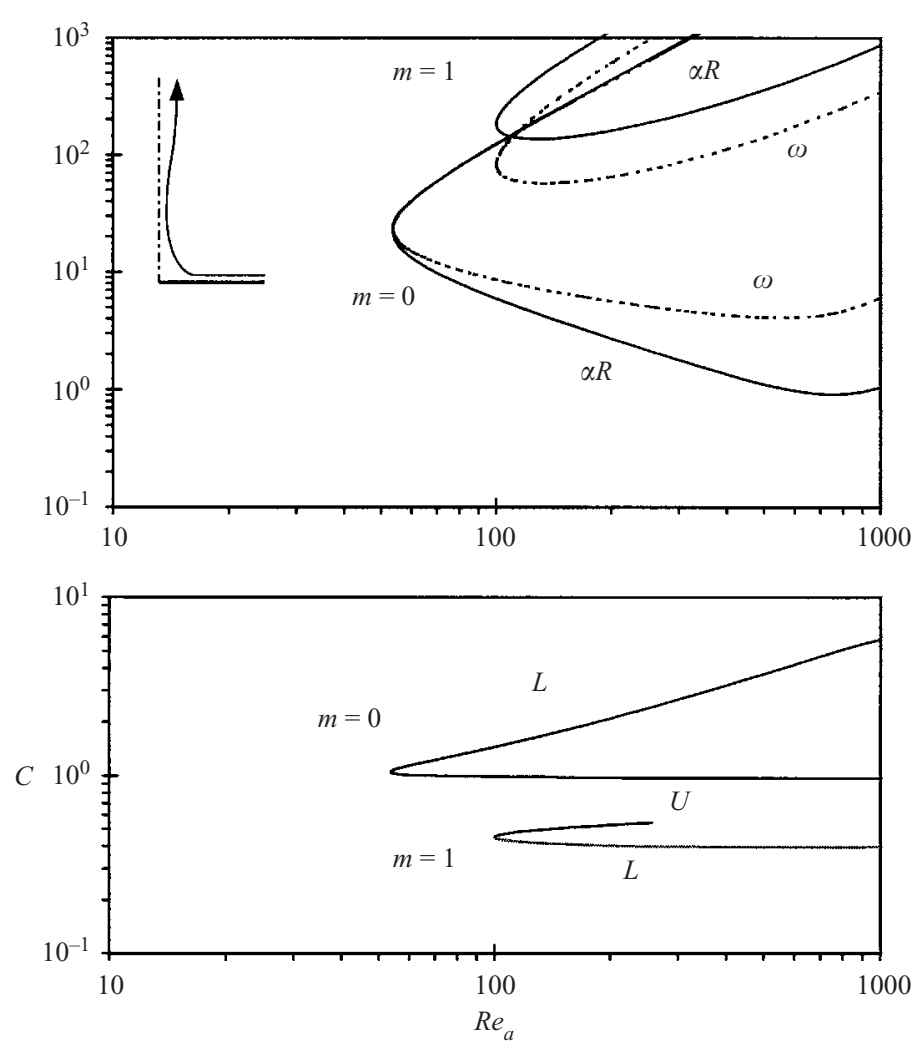

FIgURE 7. Neutral curves for the axisymmetric $(m=0)$ and helical $(m=1)$ instabilities of the Squire jet. For notation see figures 2 and 6 . The inset shows the base-flow diagram: a streamline and plane (solid), the flow direction (arrow), and the symmetry axis (dotted).

latter relation follows from the fact that $r v_{a} / \nu=\frac{1}{8} R_{M}^{2}$ for the Schlichting round jet; $v_{a}$ is the velocity on the axis). Then, our results for critical values of $R e_{D}$ are 15 for axisymmetric and 28.4 for helical disturbances.

The first experimental data reported by Batchelor \& Gill (1962) were apparently unpublished results by Schade who observed in 1958 steady laminar jets up to $R e_{D}$ of several hundred. In contrast, Viilu (1962) found that the critical $R e_{D}$ for the round jet instability is between 10.5 and 11.8. This discrepancy between Schade's and Viilu's results prompted Reynolds (1962) to study jets in the range $10<R e_{D}<300$. He observed four modes: $(a)$ puffs near the nozzle $\left(10<R e_{D}<70\right)$, (b) axisymmetric 'condensations' well away from the nozzle $\left(50<R e_{D}<200\right)$, (c) sinuous undulations of long wavelength far from the nozzle $\left(150<R e_{D}<300\right)$, and (d) formation of foot-shaped pockets of dye $\left(200<R e_{D}<300\right)$. Events $(a)$ are irrelevant for our far-field analysis, the observation of the axisymmetric instability $(b)$ at smaller $R e_{D}$ than that for the bending instability $(c)$ is consistent with our result: the critical $R e_{D}$ is smaller for axisymmetric modes than for helical.

We attempt to explain the larger values of $R e_{D}$ for the disturbances observed by Reynolds (compared with critical $R e_{D}$ predicted by our theory). Since the experiment provided no forcing of growing modes, their initial amplitude (at the nozzle exit) was very small, i.e. signal/noise ratio $\ll 1$. Therefore, the instability mode requires a significant distance to amplify up to a visually distinguishable amplitude. For slightly 
supercritical $R e_{D}$ where the spatial growth rate is small, this distance can exceed the tank length in the experiment, thus making the instability invisible. At larger $R e_{D}$, as the growth rate increases, the instability modes become visible at smaller distances from the nozzle. One could argue that this experiment revealed instability only for $R e_{D}$ much larger than the critical value.

Experiments by Mollendorf \& Gebhart (1973) show strong influence of buoyancy (even small) on the jet stability. They did not observe the axisymmetric instability, possibly because of the rather large $\operatorname{Re}_{D}$ ( $>2000$ for the lowest level of buoyancy) where transition occurs in the near field for all frequencies. We conclude that our results do not contradict experimental observations, though further experimental (with controlled disturbances) and theoretical studies (addressing non-similar flows) are required. Now we will investigate effects of a no-slip or a stress-free surface on the jet stability.

\subsection{Stability of the Squire jet}

\subsubsection{Base flow}

To examine the effect of boundary conditions on stability characteristics, we first consider a swirl-free jet in half-space (see the inset in figure $7 a$ ) that is also governed by an exact solution (Squire 1952):

$$
v_{b}=-2 R e_{p}(1-x) /\left\{b \cot \left[\frac{1}{2} b \ln (1+x)\right]-1\right\},
$$

where $b=\left(2 R e_{p}+1\right)^{1 / 2}$, and $R e_{p}$ is the Reynolds number based on the distance from the origin and the velocity on the plane, $x=0$. This follows from

$$
\begin{aligned}
u_{b} & =v_{b}^{\prime}=2 R e_{p} /\left\{b \cot \left[\frac{1}{2} b \ln (1+x)\right]-1\right\} \\
& -R e_{p}(1-x)(1+x)^{-1} b^{2}\left\{b \cos \left[\frac{1}{2} b \ln (1+x)\right]-\sin \left[\frac{1}{2} b \ln (1+x)\right]\right\}^{-2},
\end{aligned}
$$

that yields $u_{b}=-R e_{p}$ at $x=0$; the negative sign appears because we consider the velocity at the plane directed to the jet source and take its absolute value for $R e_{p}$.

Here we study the stability of this solution under either no-slip or stress-free conditions for disturbances on the plane. What conditions to use depends on a physical problem, as explained below.

Solution $(9 a)$ is relevant to flow induced by a converging motion of planar material. Such a flow can model jets developing near accretion disks in cosmic space (Shtern \& Hussain 2001). The material of the disk, moving by gravity toward a central body, drives a jet-like flow of ambient gas normal to the disk (see the inset in figure 7a). Since the disk density is much higher then the gas density, the no-slip conditions are relevant for disturbances of the gas flow.

Another application is the Marangoni convection induced by a sink of heat on the liquid surface (SH98). At zero Prandtl number, the temperature field is flowindependent and the problem reduces to a flow driven by tangential stresses given at the liquid surface. Since prescribed stresses drive the liquid, the stress-free conditions are relevant for disturbances of this flow.

For both the problems, a strong jet develops even at moderate $R e_{p}$ and the jet velocity $\left(R e_{a}\right)$ tends to infinity as $R e_{p}$ approaches 7.67 (SH98).

\subsubsection{Stability results}

Figure 7 depicts data for neutral disturbances satisfying the no-slip condition at the plane. The data for the $m=1$ helical disturbances, e.g. $R e_{a c}=100.2, \alpha_{i}=1.83$ and $\omega=82.2$, are very close to that for the Landau jet. Also, the results on helical 
instability of the Squire jet differ by less than $0.1 \%$ for the stress-free and no-slip conditions at the plane.

A physical reason for these results being so close is that neutral disturbances occupy only a region near the jet axis: the disturbance energy, $E_{d}$, rapidly decays as the polar angle increases, so that $E_{d}$ becomes negligible for $\theta>20^{\circ}$ (figure $3 a$ ). As the disturbance totally vanishes for larger $\theta$, it is not sensitive to the flow boundary location and the conditions (no-slip or stress-free) are posed there. The effective boundary condition appears to be a rapid decay of disturbance as the distance from the jet axis increases.

Thus, our results show that the helical instability occurs within the near-axis boundary layer, i.e. inside the Schlichting jet developing for large $R e_{a}$ in the both Landau and Squire solutions. This supports our view that our results and the parallelflow-theory results for helical instability differ owing to deceleration, and not because of the boundary-layer approximation of the base flow.

The difference in stability results for axisymmetric disturbances of the Landau and Squire jets is more remarkable: $R e_{a c}=53.07, \alpha_{i}=0.417$ and $\omega=23.5$ (noslip); $R e_{a c}=48.4, \alpha_{i}=0.323$ and $\omega=16.2$ (stress-free); while for the Landau jet, $R e_{a c}=28.1, \alpha_{i}=0.097$ and $\omega=2.71$. So these large-scale (i.e. small $\alpha_{i}$ ) axisymmetric disturbances appear rather sensitive (in contrast to helical modes) to the difference in the boundary conditions and in the flow region. To study this dependence in more detail, we now consider flows inside a cone.

\subsection{Stability of a jet in a cone}

\subsubsection{Base flow}

The Squire solution is easily generalized for a conical flow region, $x_{c} \leqslant x \leqslant 1$ :

$$
v_{b}=-\operatorname{Re}_{p}\left(1+x_{c}\right)\left(1-x_{c}\right)^{-1}(1-x)\left\{b \cot \left(\frac{1}{2} b \ln \left[(1+x) /\left(1+x_{c}\right)\right]-1\right\}^{-1},\right.
$$

where $b=\left[2 \operatorname{Re}_{p}\left(1+x_{c}\right)\left(1-x_{c}\right)^{-1}+1\right]^{1 / 2}$ and $u_{b}=-\operatorname{Re} e_{p}$ at $x=x_{c}$.

This solution is a model of a capillary flow in a conical liquid meniscus in electrosprays (Shtern \& Barrero 1995).

\subsubsection{Stability results}

Figure 8 shows the critical value of $R e_{a}$ versus the cone angle $\theta_{c}\left(x_{c}=\cos \theta_{c}\right)$. The results include those for the Squire $\left(x_{c}=0\right)$ and Landau $\left(x_{c}=-1\right)$ jets as well. The solid curves correspond to the stress-free condition on the cone surface, $x=x_{c}$, and the dashed curves are for no-slip. The numbers near curves indicate the $m$ value and curve $0 s$ is for the axisymmetric steady-state instability causing the appearance of swirl in liquid cones (Shtern \& Barrero 1995). Our calculations show that the minimum $R e_{a}$ for this mode occurs at $\omega=0$ and $\alpha_{i}=0$, so this instability is not oscillatory either in time or in space.

The $m=1$ curves for the no-slip and stress-free conditions coincide within the accuracy of the drawing in figure 8 , while the $m=0$ curves are distinct although close. These features confirm the boundary-layer character of the $m=1$ instability and the global character of the axisymmetric instability.

We found no instability of these flows with respect to disturbances with $m>1$ for $R e_{a}>0$ (in oppositely directed flows where $R e_{a}<0$, the $m>1$ instability does occur, e.g. see SH98). In contrast, the $|m| \geqslant 1$ instability is typical for swirling jets, as we show below. 


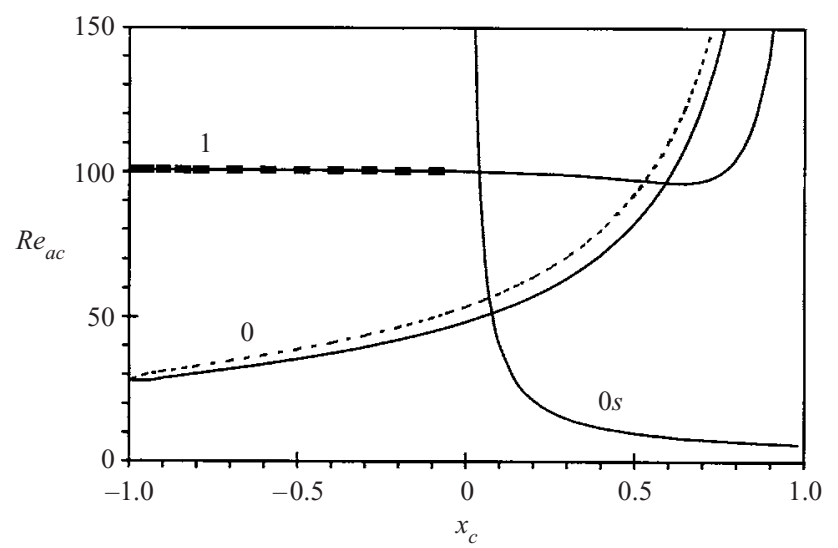

FIGURE 8. Dependence of the critical Reynolds number $R e_{a}$ on the cone angle for swirl-free jets inside a cone of angle $\theta_{c}\left(x_{c}=\cos \theta_{c}\right)$. - , stress-free; ---, no-slip condition. Numbers near the curves show the azimuthal wavenumber $m$. Curve $0 s$ is for the steady-state instability leading to the appearance of swirl.

\section{Stability of swirling jets}

\subsection{Base flow}

Consider a swirling flow induced by a half-line vortex located on the negative $z$-axis, i.e. at $x=-1$. Such a flow can model a tornado or a leading-edge vortex (Shtern \& Drazin 2000). These flows can expand abruptly in a wide-angle cone (wake of vortex breakdown). The half-line singularity mimics a consolidated vortex core upstream of vortex breakdown.

The flow characteristics are the vortex circulation and the flow force $J$ acting on a surface surrounding the singularity. Since there is no source of momentum outside the half-line vortex, the surface can be chosen rather arbitrarily. For example, the plane, $z=z_{0}>0$, is an appropriate choice which Long (1961) applied to the near-axis boundary layer. The corresponding dimensionless parameters are the swirl Reynolds number $R e_{s}=\Gamma_{b}(-1)$ and $J_{0}=J /\left(2 \pi \rho \nu^{2}\right)$ or Long's parameter $M=2 \pi J_{0} / R e_{s}^{2}$.

In contrast to swirl-free jets studied in $\S 3$, the base flow for swirling jets has no analytical solution, except for some limiting cases. So, together with integration of the stability problem, we must numerically calculate the base flow as well. Referring to Shtern \& Hussain (1996) for details, we show here only the governing equations,

$$
\begin{gathered}
\left(1-x^{2}\right) \Psi^{\prime}+2 x \Psi-\frac{1}{2} \Psi^{2}=F, \\
\left(1-x^{2}\right) F^{\prime \prime}+2 x F^{\prime}-2 F=\Gamma_{b}^{2}, \\
\left(1-x^{2}\right) \Gamma_{b}^{\prime \prime}=\Psi \Gamma_{b}^{\prime},
\end{gathered}
$$

the boundary conditions,

$$
\Psi(1)=\Gamma_{b}(1)=0, \quad \Psi(-1)=0, \quad \Gamma_{b}(-1)=R e_{s},
$$

and the integral condition,

$$
\int_{0}^{1}\left\{x\left(2-\Psi^{\prime}\right) \Psi^{\prime}-x\left[(2-\Psi) \Psi-x F^{\prime}\right] /\left(1-x^{2}\right)-F^{\prime}\right\} \mathrm{d} x=J_{0},
$$




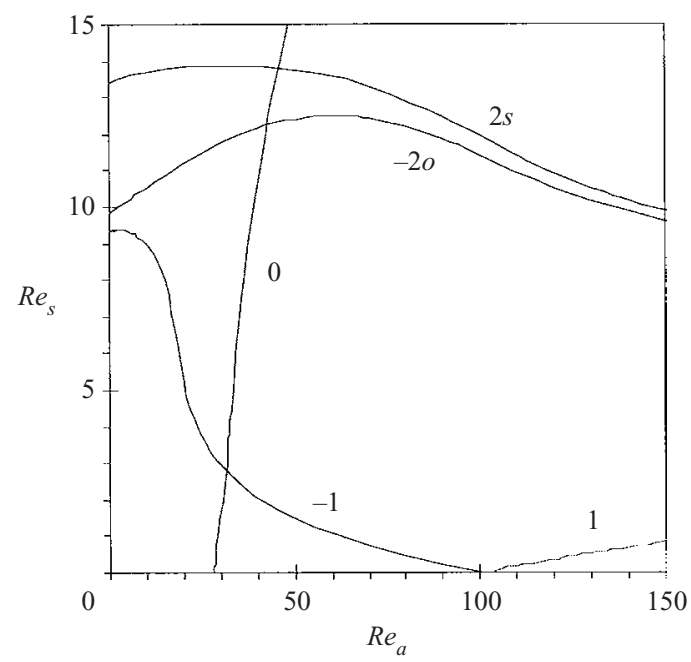

FIGURE 9. Critical swirl Reynolds number $R e_{s} v s$. the axial Reynolds number $R e_{a}$ for one-cell swirling flows. Numbers near curves indicate the azimuthal wavenumber $m$. $o$, time-oscillating and $s$, steady neutral modes.

used for the numerical integration of the base flow. In (10), $\Psi=-v_{b}, \Psi^{\prime}=-u_{b}, F$ is an auxiliary function replacing pressure, $p_{b}=\left(2 x \Psi-F^{\prime}-\Psi^{2}\right) /\left(1-x^{2}\right)$, and the prime denotes differentiation with respect to $x$.

Depending on $R e_{s}$ and $J_{0}$, the flow has a single cell where the fluid goes from $z=-\infty$ to $z=\infty$ or two cells where the fluid goes from both $z=-\infty$ and $z=\infty$ toward the coordinate origin along the axis and then away from the origin along a conical surface, $x=x_{s}$, which separates the flow cells. As $R e_{s} \rightarrow \infty$, a boundary layer can develop near the positive $z$-axis, $x=1$; the flow reduces to Long's vortex in this region. However, the boundary layer can develop near $x=x_{s}$ as well (i.e. away from the axis); this high- $R e_{s}$ flow is not described by Long's approximation.

An important feature is that three different solutions can exist at the same values of $R e_{s}$ and $J_{0}$, comprising a hysteresis loop (Shtern \& Hussain 1996), so these parameters do not uniquely specify the flow (e.g. curve $a$ in figure $1 a$ ). For this reason, we use either $R e_{a}=u_{b}(1)$ or $x_{s}$ as a control parameter instead of $J_{0}$ because $R e_{a}$ uniquely specifies the one-cell flow where $R e_{a} \geqslant 0$ and $x_{s}$ uniquely specifies the two-cell flow (where $R e_{a}<0$ ).

Shtern \& Drazin (2000) studied the spatial stability of this flow to time-monotonic (zero-frequency) disturbances. Here, we consider generic (time-oscillating, threedimensional) disturbances and reveal new important features.

\subsection{Stability of one-cell flows}

Figure 9 shows the dependence between the swirl Reynolds number $\operatorname{Re}_{s}$ and the axial Reynolds number $R_{a}$ (based on the velocity on the axis, $x=1$ ) at the critical neutral points corresponding either to the minimum $R e_{a}$ at fixed $R e_{s}$ (for curves 0 , 1 and -1$)$ or to the minimum $R e_{s}$ at fixed $R e_{a}$ (for curves $(-2 o, 2 s$ and -1$)$. The numbers near the curves indicate values of the azimuthal wavenumber $m$, and ' $o$ ' and ' $s$ ' denote oscillating and steady neutral disturbances, respectively. Curve $2 s$ is taken from Shtern \& Drazin (2000) for comparison whereas the other curves are new; the comparison shows that time-oscillatory disturbances are more dangerous 
than time-monotonic (as curve $-2 o$ lies below curve $2 s$ ). The flows are stable in the region located below the $m=-1$ curve and to the left of the $m=0$ curve.

The points of the curves 1,0 and -1 located on the abscissa $\left(R e_{s}=0\right)$ in figure 9 correspond to the critical values of $R e_{a}$ for the Landau jet. The curves 1 and -1 merge at $R e_{s}=0$ because $R e_{a c}$ is independent of the sign of $m$ for swirl-free flows. Swirl breaks this symmetry and the curves 1 and -1 rapidly diverge as $R e_{s}$ increases. Critical $R e_{a}$ is smaller for the $m=-1$ mode compared with the $m=1$ mode at the same value of $R e_{s}$.

One possible reason for this feature is in the orientation of the disturbance $\left(\omega_{d}\right)$ and base-flow $\left(\omega_{b}\right)$ vorticity vectors. For the Kelvin-Helmholtz instability, these vectors are parallel for the most growing disturbance (as discussed in $\S 3.1 .2$ ). We have calculated the vorticity components for disturbances,

$\omega_{d r}=-\Gamma_{d x}-v_{d \phi} /\left(1-x^{2}\right), \quad \omega_{d \theta}=\left(u_{d \phi}-\Gamma_{d \xi}\right) / \sin \theta, \quad \omega_{d \phi}=\left[v_{d \xi}+\left(1-x^{2}\right) u_{d x}\right] / \sin \theta$,

and for the base flow,

$$
\omega_{b r}=-\Gamma_{b}^{\prime}, \quad \omega_{b \theta}=0, \quad \omega_{b \phi}=-\left(1-x^{2}\right) \psi^{\prime \prime} / \sin \theta
$$

(scaled by $v / r^{2}$ ) at the $\theta$ value where $\left|\omega_{b}\right|$ has its maximum, and have found that the angle between $\omega_{d}$ and $\omega_{b}$ is smaller for the $m=-1$ mode than for the $m=1$ mode. As the angle decreases, the growth rate increases, thus explaining why counter-rotating modes are more dangerous than co-rotating. This reason is valid for parallel flows as well.

Since $R e_{s}<10$ and $R e_{a}<32$ for stable flows, their boundary-layer approximation is questionable. Recall that Long's Type II solution corresponds to the intermediate branch of the hysteresis loop (Shtern \& Hussain 1996) which develops only for $R e_{s}>R e_{s c u}=11.5$ (figure $1 a$ ). For $R e_{s}<R e_{s c u}$, where the instability occurs, there is no hysteresis and, therefore, no Long's Type II solution. Therefore, this solution either does not exist (for $R e_{s}<R e_{s c u}$ ) or is unstable (for $R e_{s}>R e_{s c u}$ ). We conclude that our results are stronger than previous results on the stability of Long's Type II solution, obtained using the boundary-layer approximation (i.e. for $R e_{s} \gg R e_{s c u}$ ).

Long's Type II solution includes annular jets where the location of the maximum axial velocity (at a fixed $r$ ) is shifted away from the axis of symmetry. In contrast to Long's Type II solution, annular swirling flows exist for any small $R e_{s}$ as well (see the $R e_{a}<0$ region in figure $1 a$ ). To understand their instability character better, we now consider in more detail the annular flow with zero velocity on the axis $\left(R e_{a}=0\right.$, see the inset in figure $10 c$ ).

Figure 10 shows neutral curves at $R e_{a}=0$. An important new feature is the character of the $m=-2$ instability. The phase velocity, $C=\omega /\left(\alpha_{i} v_{\text {rmax }}\right)$ is negative on most of the $m=-2$ neutral curve (figure 10c) including the vicinity of the $R e_{s}$ minimum. In addition, this double-helix mode rotates in the positive- $\phi$ direction, i.e. in the same direction as the base flow (co-rotation), while the single-helix mode is counter-rotational.

The $m=-2$ neutral curves in figures $10(b)$ and $10(c)$ intersect the lines $\omega=0$ and $C=0$ as $\alpha_{i}$ increases along the upper branch of the $m=-2$ neutral curve in figure $10(a)$ (these intersection points comprise curve $2 s$ in figure 9). Thus, short-wave modes are counter-rotational and have positive phase velocity whereas long doublehelix waves are co-rotational and have negative phase velocity. As discussed in $\S 3.1 .2$, short waves are less sensitive to the flow non-parallelism and satisfy the condition, $0<C<1$, which is valid for disturbances in parallel flows. In contrast, the phase 

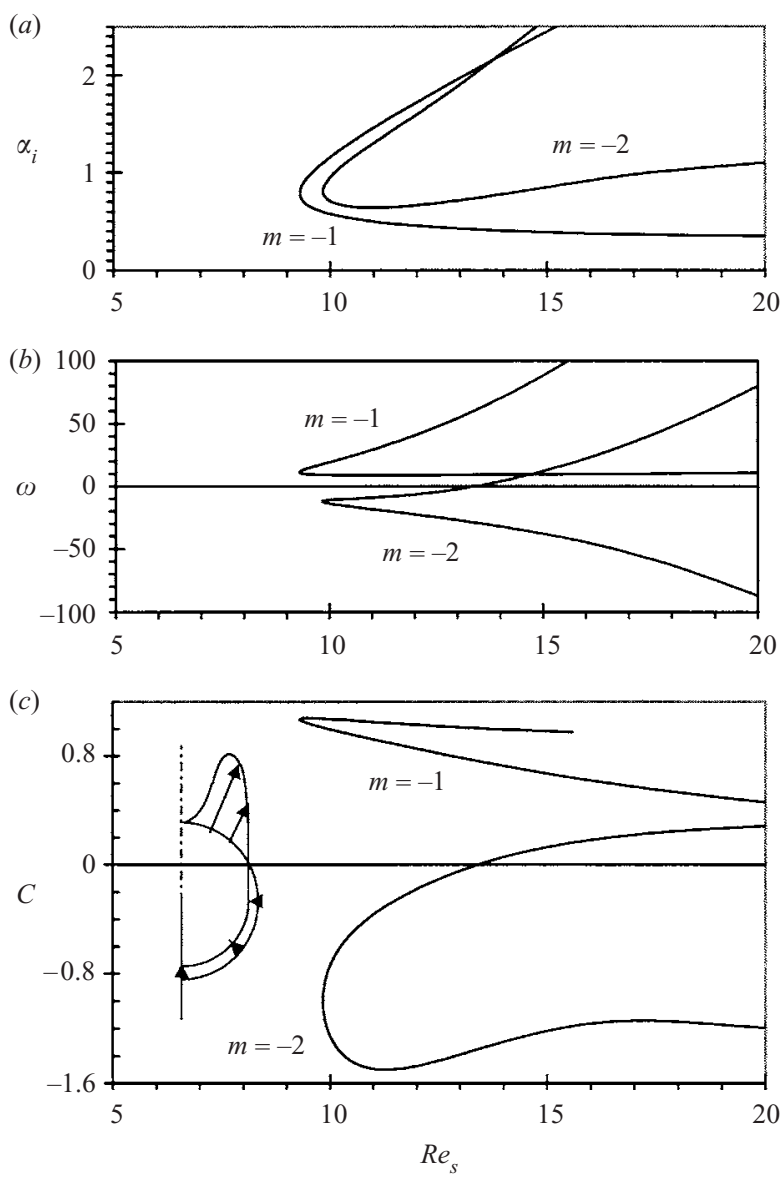

FIGURE 10. Neutral curves of the annular swirling jet at $R e_{a}=0$ (zero velocity on the axis; see inset) for helical disturbances with the azimuthal wavenumber $m=-1$ and $m=-2$. Dependence of $(a)$ the radial wavenumber $\alpha_{i},(b)$ frequency $\omega$, and $(c)$ phase velocity $C$ on swirl Reynolds number $R e_{s}$. The inset in $(c)$ sketches the dependence of the base-flow radial velocity on the polar angle.

speed of long waves can be out of this range: $C>1$ for long axisymmetric waves (§3.1.2) and $C<0$ for the $m=-2$ long waves (figure $10 c$ ).

Let us attempt now to interpret these results for vortex breakdown. The first and the most popular explanation of axisymmetric (bubble-like) vortex breakdown is in terms of long standing waves (Squire 1956; Benjamin 1962; Keller, Egli \& Exley 1985). An alternative view is that axisymmetric vortex breakdown is a flow separation from the axis, rather than a wave or an instability effect (Hall 1972; Goldshtik \& Hussain 1998). Gelfgat et al. (1996) studying stability of a confined swirling flow found that the instability and the development of a separation zone (vortex-breakdown bubble) are different phenomena. Our results are in favour of the separation scenario for open flows as well. Indeed, we have shown that the axisymmetric instability is oscillatory, so that no standing axisymmetric wave occurs in swirling jets. In addition, Shtern \& Drazin (2000) showed that the flow reversal (i.e. appearance of a separation zone) occurs without instability. These two results together support the view that the bubble-like vortex breakdown is a flow separation. 


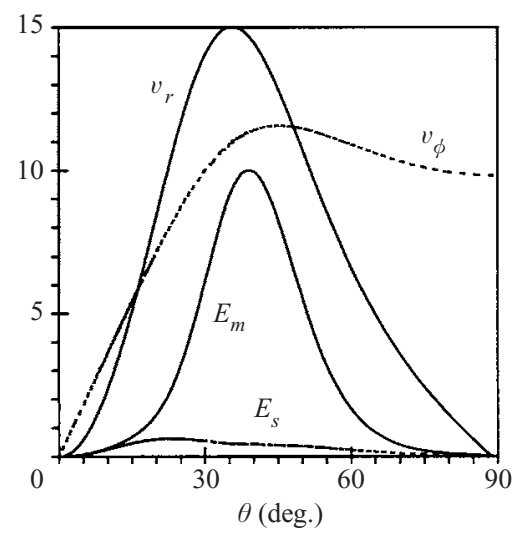

FiguRE 11. Dependence of the radial $v_{r}$ and swirl $v_{\phi}$ velocities as well as of the meridional $E_{m}$ and swirl $E_{s}$ kinetic energies of the $m=-2$ neutral disturbances on the polar angle $\theta$ at critical $R e_{s}$ for the annular jet with $R e_{a}=0$ (figure 10).

In contrast, the helical vortex breakdown undoubtedly is an instability effect because it breaks the axisymmetry of the upstream flow (any symmetry breaking is a result of instability, i.e. of a growing disturbance transforming the symmetric state into an asymmetric one).

Being long in the radial direction, the $m=-2$ mode has also a wide extent in the $\theta$ direction (cf. figure 11 with figures 3 and 4). Figure 11 also shows that the disturbance energy of the swirl motion, $E_{s}=\left|\Gamma_{d}\right|^{2} /\left(1-x^{2}\right)$, is very small compared with that of the meridional motion, $E_{m}=\left|u_{d}\right|^{2}+\left|v_{d}\right|^{2} /\left(1-x^{2}\right)$, i.e. the instability affects mainly the meridional motion. This fact indicates that the double-helix instability results from the radial divergence of streamlines (significantly enhanced by swirl) rather than being due to the shear of the base flow. The results of the following section support our view that this instability is of the divergent type.

\subsection{Stability of two-cell flows}

Figure 12 shows the critical $R e_{s}$ versus the angle $\theta_{s}$ of conical surface separating the flow cells $\left(x_{s}=\cos \theta_{s}\right)$. The numbers near the curves indicate values of $m$, and the letters ' $o$ ' and ' $s$ ' denote oscillating and steady neutral modes. Figure 12 does not depict neutral curves for $|m|>2$ because their critical $R e_{s}$ are larger than those for the $|m| \leqslant 2$ modes. The $m=-1$ helical mode appears to be the most dangerous (i.e. corresponding to the smallest critical $R e_{s}$ ) in the entire range, $-1<x_{s} \leqslant 1$.

The $m= \pm 2$ modes are also of physical interest because critical $R e_{s}$ values are close for $m= \pm 2$ and $m=-1$ modes and these modes can interact (in the nonlinear development of instability). For small separation angles $\theta_{s}$ (i.e. for $x_{s}$ close to 1 ), only the $m=-2$ mode can be neutral or growing while the $m=2$ disturbances decay. As decreasing $x_{s}$ passes through $x_{s}=0.9$, a new important effect occurs: a neutral mode with $\alpha_{i}=0$ appears. Along the $\alpha_{i}=0$ curve in figure 12 , the $R e_{s}$ value goes to infinity as increasing $x_{s}$ approaches 0.9 ; the $\alpha_{i}=0$ neutral disturbance does not exist for $x_{s}>0.9$. The $\alpha_{i}=0$ disturbances oscillate in phase along rays $\phi=$ const.

Changing the sign of $\alpha_{i}$ is equivalent to changing the sign of $m$. Indeed, the stability problem is invariant under the transformation $\left\{m \rightarrow m, \alpha_{i} \rightarrow-\alpha_{i}, \omega \rightarrow-\omega, \Gamma_{d} \rightarrow\right.$ $-\Gamma_{d}$, and complex conjugation $\}$ according to (5), so that it is sufficient to consider only positive $\alpha_{i}$. When decreasing $\alpha_{i}$ passes through 0 along a neutral curve, this is 


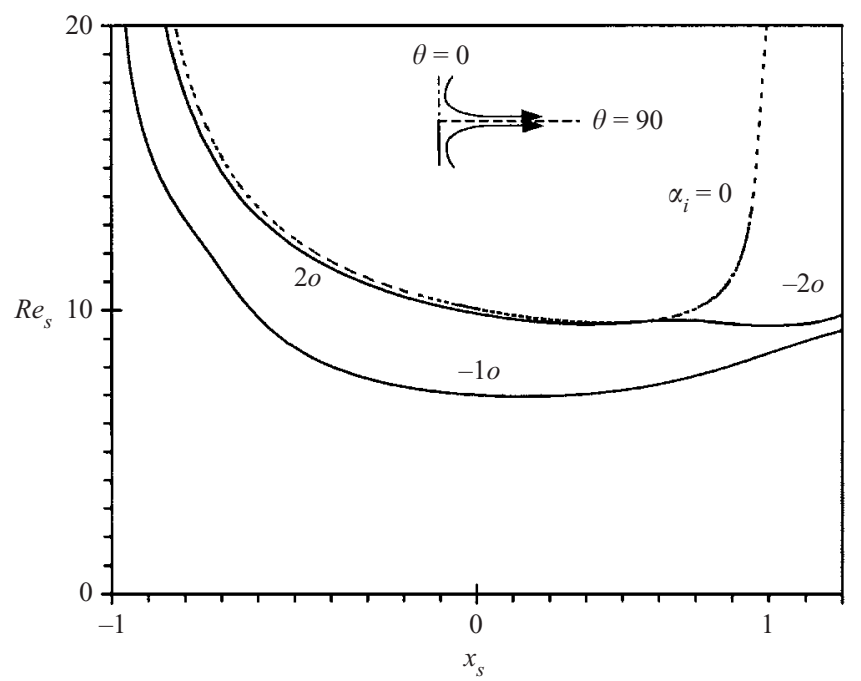

FIGURE 12. Critical value of swirl Reynolds number $R e_{s} v s$. the separation angle $\theta_{s}\left(x_{s}=\cos \theta_{s}\right)$ for two-cell flows. Numbers near curves denote values of $m ; o$, oscillating and $s$, steady neutral modes; the dashed curve $\left(\alpha_{i}=0\right)$ is for standing-wave oscillations. The point, where curves $2 o$ and $-2 o$ touch the $\alpha_{i}=0$ curve, separates curves $2 o$ and $-2 o$. The inset shows a flow diagram: streamlines and singularity half-line (solid), the flow direction (arrows), separating line (dashed), and the symmetry axis (dotted).

equivalent to the appearance of a neutral mode with $\alpha_{i}>0$ for the opposite sign of the azimuthal wavenumber $m$. The neutral curve $\alpha_{i}\left(R e_{s}\right)$ for negative $m$ is the reflection with respect to line $\alpha_{i}=0$ of the curve $\alpha_{i}\left(R e_{s}\right)$ for positive $m$. Figure 13 shows (at $x_{s}=0$ ) such neutral curves along which $\alpha_{i}$ passes through zero.

The base flow at $x_{s}=0$ is a swirling jet spiralling out along the equatorial plane, $x=0$, as the inset in figure 13(a) depicts. Figure 13 presents only positive values of $\alpha_{i}$ and $\omega$ because the results are symmetric to the transformation $\left\{m \rightarrow-m, \alpha_{i} \rightarrow\right.$ $\left.-\alpha_{i}, \omega \rightarrow-\omega\right\}$. In figure 13(a), the neutral curves for $m=2$ and $m=-2$ intersect at $\alpha_{i}=0$ and $R e_{s}=10.05$ (which is slightly larger than the critical value, $R e_{s c}=9.87$ ). At $\alpha_{i}=0$, the disturbance is proportional to $\exp [\mathrm{i}(m \phi-\omega \tau)]$ with no oscillation in the $r$-direction. Since $\omega>0$ for $m=2$ at $\alpha_{i}=0$, this mode rotates in the positive- $\phi$ direction (co-rotation: $\mathrm{d} \phi / \mathrm{d} \tau=\omega / m>0$ ), as also does the neutral disturbance at $R e_{s}=R e_{s c}$. The $m=-2$ neutral solution at $\alpha_{i}=0$ is the complex conjugate of the $m=2$ solution, therefore both the solutions describe the same mode.

For $\alpha_{i}>0$, the $m=2$ curve has smaller $R e_{s}$ compared with the $m=-2$ curve (figure $13 a$ ), i.e. the $m=2$ mode is more dangerous than the $m=-2$ mode. Since along the $m=-2$ curve in the range $\alpha_{i} \geqslant 0, \operatorname{Re}_{s}$ reaches its minimum value at $\alpha_{i}=0$, this minimum can be interpreted as the critical $R e_{s}$ for the $m=-2$ mode.

As increasing $x_{s}$ passes through $x_{s}=0.43$ (where the $\alpha_{i}=0$ curve touches the $m=2$ and $m=-2$ curves in figure 12), the $m=-2$ mode becomes more dangerous than the $m=2$ mode. So the $\alpha_{i}=0$ curve serves as the continuation of the curve $-2 o$ for $x_{s}<0.43$ and as the continuation of the curve $2 o$ for $x_{s}>0.43$ in figure 12 . As increasing $x_{s}$ approaches $x_{s}=0.9, R e_{s}$ goes to infinity along the $\alpha_{i}=0$ curve. This fact indicates that there is no instability to the $m=2$ disturbances for $x_{s}>0.9$ that agrees with the results for $x_{s}=1$ shown in figure 10 , where the $m=-2$ mode is only responsible for the double-helix instability. 

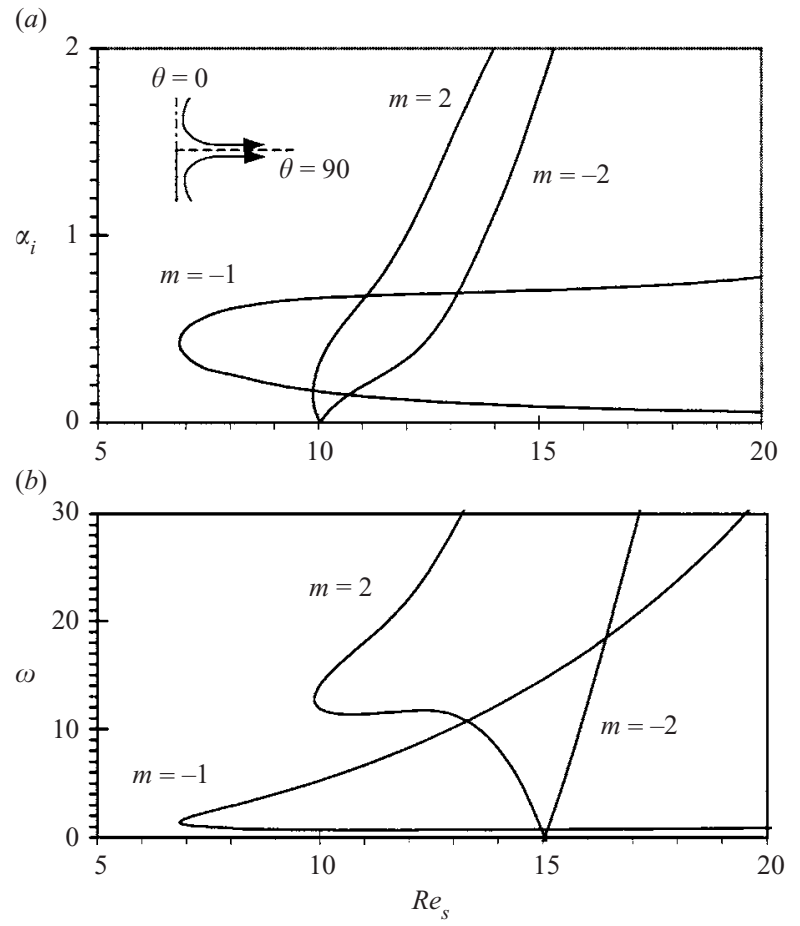

FiguRE 13. Neutral values of $(a)$ radial wavenumber $\alpha_{i}$ and $(b)$ frequency $\omega$ vs. swirl Reynolds number $\operatorname{Re}_{s}$ for a swirling jet spiralling out along the equatorial plane, $\theta=90^{\circ}$ (inset sketches the meridional flow). Azimuthal wavenumbers $m=-1$ and $m= \pm 2$ characterize the most dangerous modes.

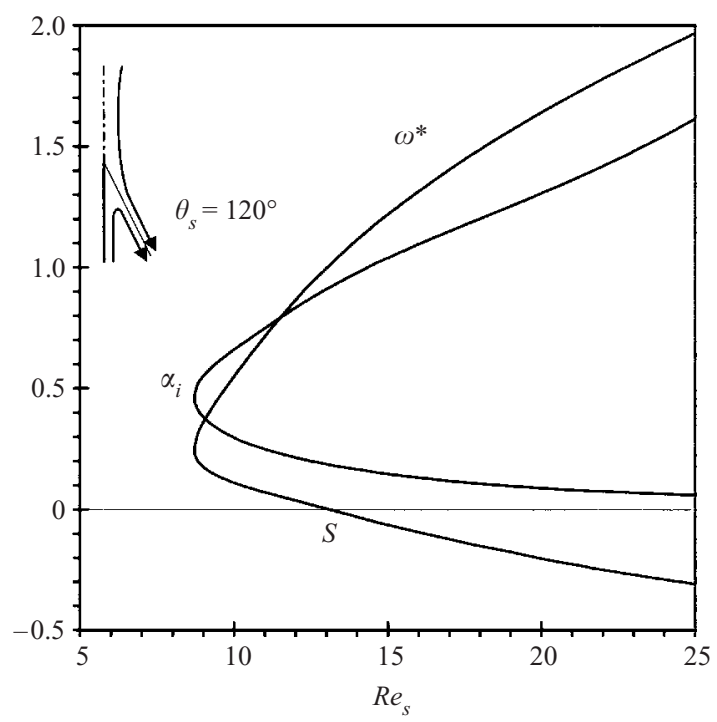

FiguRE 14. Neutral curve for single-helix disturbances of two-cell flow with $x_{s}=-0.5$ $\left(\theta_{s}=120^{\circ}\right.$; inset sketches the meridional flow). Steady-state instability (at $\left.\omega=0\right)$ is marked by $S ; \omega^{*} \equiv \omega /[1+|\omega| / \log (1+|\omega|)]$. 
For $x_{s}<-0.2$, the phase velocity, $c$, changes its sign for single-helix disturbances as well. Figure 14 shows this feature for a two-cell flow with $x_{s}=-0.5$ (see the inset). We rescale frequency, $\omega^{*}=\omega /[1+|\omega| / \log (1+|\omega|)]$, to plot the dependences of wavenumber $\alpha_{i}$ and frequency $\omega$ on $R e_{s}$ in one figure. The frequency passes through zero at point $S$ that corresponds to the steady-state instability. For smaller $R e_{s}, c$ is positive and for larger $R e_{s}$, there are disturbances with negative $c$. For all swirling flows considered here, the oscillatory instability is more dangerous that the steady-state instability studied by Shtern \& Drazin (2000).

A common feature for all separation angles of the two-cell flow is that the meridional-motion part $E_{m}$ of the disturbance kinetic energy is significantly larger than the swirl-motion part $E_{s}$ for critical disturbances. We interpret this fact that the instability results from the radial divergence of streamlines (provided by swirl), but not from the direct effect of the swirl. Indeed, swirl-free flows with the radial divergence of streamlines are also unstable to azimuthal modes ( $\mathrm{SH} 98)$ (the simplest example of the divergent instability occurs in the planar source flow which is shearfree; Goldshtik et al. 1991) and this instability (occurring for arbitrarily large $m$ as Reynolds number increases) is very similar to that for the swirling jets. Physical reasons for this similarity are discussed in more detail below.

\section{Concluding discussion}

Our results differ significantly from known results on instability of round jets in two major aspects: (i) swirl-free conical jets are unstable to axisymmetric disturbances (prior studies missed this instability), and (ii) the helical instability of both swirl-free and swirling jets occurs for smaller Reynolds numbers than those predicted by quasiparallel or boundary-layer approximations. These differences stem from the strongly non-parallel character of the flow - a feature properly accounted for by the approach developed here.

This approach exploits the fact that the base flows, being conically similar, are far-field approximations of practical flows, permitting a helpful transformation of variables and justifying the far-field approximation for disturbances. As a result, we reduce the linear stability problem to ordinary differential equations for these strongly non-parallel flows.

This reduction permits a detailed investigation of the flow stability using neither boundary-layer nor parallel-flow approximations. This investigation reveals two important features of non-parallel flows, which significantly affect their stability: $(A)$ deceleration that increases the growth rate of the shear-layer instability and $(B)$ swirlinduced wide divergence of streamlines that causes an additional - divergent instability occurring even in shear-free flows. The boundary-layer and quasi-parallel approximations fail to account for both these features adequately. Although the parallel theory predicts that decelerating flows are less stable than accelerating (e.g. for the Falkner-Scan boundary layers), this prediction is based only on the difference in velocity profiles, particularly, the appearance of the inflection point in the profile of the decelerating flow, which is a side effect of the flow deceleration.

Unfortunately, the parallel-flow (as well as boundary-layer) approximation misses the direct destabilizing effect of the base-flow deceleration. The term responsible for this destabilizing effect is $v_{d s} \partial v_{b s} / \partial s$ ( $s$ denotes the streamwise coordinate and velocity component while $d$ and $b$ mark the disturbance and base velocities), as follows from the streamwise momentum equation,

$$
\partial v_{d s} / \partial t+v_{b s} \partial v_{d s} / \partial s=-v_{d s} \partial v_{b s} / \partial s+\text { other terms. }
$$


The first term in the right-hand side (neglected in the parallel-flow theory) contributes to the disturbance growth rate positively when the base flow decelerates $\left(\partial v_{b s} / \partial s<0\right)$ and negatively when the base flow accelerates $\left(\partial v_{b s} / \partial s>0\right)$. Figure 4 provides a diagram of this effect.

The parallel-flow theory misses and our approach accounts for this destabilizing effect of deceleration; that explains the difference in the stability results. The difference is expected to be more prominent for large-scale disturbances than for small-scale ones. Indeed, $\partial v_{d s} / \partial s$ increases with the streamwise wavenumber while $\partial v_{b s} / \partial s$ does not. Therefore, $v_{b s} \partial v_{d s} / \partial s$ dominates $v_{d s} \partial v_{b s} / \partial s$ for short waves and this fact diminishes the destabilizing effect of the base-flow deceleration.

Our results for swirl-free jets agree well with this expectation: the axisymmetric neutral mode has a larger wavelength than the helical mode and the more intense stretching of their vorticity (as for vortex rings). Accordingly, the difference between our and the parallel-flow results is more significant for axisymmetric disturbances. The parallel-flow theory predicts no axisymmetric instability whereas our approach reveals that this instability does occur. Moreover, it is even more dangerous than the helical instability. In contrast to this qualitative mismatch in axisymmetric instability, the results differ only quantitatively (parallel-flow $R e_{a c}$ is nearly twice our $R e_{a c}$ ) for helical instability.

The physical reason for axisymmetric disturbances being more dangerous than helical ones in swirl-free jets is probably due to the combined effects of the shear-layer (Kelvin-Helmholtz) instability and flow deceleration. The role of shear is clear from the equation for disturbance kinetic energy $E_{d}: \partial E_{d} / \partial t=-v_{d r} v_{d \theta} r^{-1} \partial v_{b r} / \partial \theta+$ other terms. The first term on the right-hand side being positive causes $E_{d}$ to grow. The vortex-dynamics mechanism of this instability (Batchelor 1967, p. 515) shows that a wavy disturbance of a vortex sheet in the plane normal to the base-flow vorticity has a positive feedback: progressive accumulation of vorticity in clumps causes the perturbation to grow. Since a spanwise disturbance has no positive feedback, a twodimensional mode is more dangerous than a three-dimensional mode of the same magnitude of wave vector.

For swirl-free jets, the base-flow deceleration just enhances the shear-layer (KelvinHelmholtz) instability. The shear-layer character of this instability is apparent from the fact that the neutral disturbances occupy only the high-shear flow region near the inflection point of the base velocity profile and vanish away from this region (distributions of critical-disturbance energy and of the base-flow shear nearly coincide). This shear-layer instability induces travelling-wave neutral modes and is limited to disturbances with the azimuthal wavenumber $m=0$ and $m= \pm 1$ only.

The other non-parallel factor - strong divergence of streamlines - leads to an additional divergent instability that occurs even without shear, e.g. in a planar source flow (Goldshtik et al. 1991). In contrast to the shear-layer instability, the divergent instability causes the growth of modes with arbitrarily large $m$ as the Reynolds number increases.

For the flows studied here, the strong divergence of streamlines results from the centrifugal effect of swirl that pushes the fluid away from the axis. This swirl-induced divergence makes a difference: the divergent instability of swirl-free flows (e.g. the planar source flow) is symmetric with respect to the sign of $m$ whereas swirl breaks this symmetry; counter-rotating $(m<0)$ disturbances are typically more dangerous than co-rotating $(m>0)$ ones. Swirl breaks this symmetry not only for the divergent but also for shear-layer instability (see curves 1 and -1 in figure 9 showing that the $m=-1$ instability is more dangerous that the $m=1$ instability, even for weak swirl). 
The angle between vectors of the base-flow and disturbance vorticity is smaller for the $m=-1$ mode than for the $m=1$ mode. This agrees with the Kelvin-Helmholtz mechanism where the most-growing-disturbance vorticity and the base-flow vorticity are parallel.

Our results also show that the shear-layer instability of conical jets is oscillatory whereas the divergent instability involves steady-state (zero frequency) modes as well. The critical Reynolds numbers for both the divergent and shear-layer instabilities are here so small that the boundary-layer approach is invalid. In particular, the Long's Type II boundary-layer solution disappears for critical values of $R e_{s}(<10$ which is less than the cusp $R e_{s}=11.5$ ), whereas its stability features have been much studied.

An effect occurring in two-cell swirling flows is the existence of precession modes. These disturbances have $\alpha_{i}=0$ (see figure 12; $\alpha_{i}$ is the radial wavenumber) and counter-rotates or co-rotates with respect to the base-flow swirl (depending on the separation angle of the two-cell flow). This might help to explain the development of jet precession in combustion chambers (Nathan, Hill \& Luxton 1998).

Thus, our study has revealed new important stability features of strongly nonparallel swirl-free and swirling jets.

\section{REFERENCES}

Ardalan, K., Draper, K. \& Foster, M. R. 1995 Instabilities of the Type I Long's vortex at large flow force. Phys. Fluids 7, 365-373.

Batchelor, G. K. 1967 An Introduction to Fluid Dynamics. Cambridge University Press.

Batchelor, G. K. \& Gill, A. E. 1962 Analysis of the stability of axisymmetric jets. J. Fluid Mech. 14, 529-551.

Benjamin, T. B. 1962 Theory of vortex breakdown phenomena. J. Fluid Mech. 14, 593-629.

Drazin, P. G., Banks, W. H. H. \& Zaturska, M. B. 1995 The development of Long's vortex. J. Fluid Mech. 286, 359-377.

Drazin, P. G. \& ReID, W. H. 1981 Hydrodynamic Stability. Cambridge University Press.

FERnANDEZ-FerIa, R. 1996 Viscous and inviscid instabilities of non-parallel self-similar axisymmetric vortex cores. J. Fluid Mech. 323, 339-365.

Fernandez-Feria, R. 1999 Nonparallel stability analysis of Long vortex. Phys. Fluids 11, 1114-1126.

Foster, M. R. \& Duck, P. W. 1982 The inviscid stability of Long's vortex. Phys. Fluids 25, $1715-1718$.

Foster, M. R. \& JACKmin, F. T. 1992 Non-parallel effects in the stability of Long's vortex. J. Fluid Mech. 244, 289-306.

Foster, M. R. \& Smith, F. T. 1989 Stability of Long's vortex at large flow force. J. Fluid Mech. 280, 405-432.

Gelfgat, A. Y., Bar-Yoseph, P. Z. \& Solan, A. 1996 Stability of a confined swirling flow with and without vortex breakdown. J. Fluid Mech. 311, 1-36.

Goldshtik, M. A. \& Hussain, F. 1998 Analysis of inviscid vortex breakdown in a semi-infinite pipe. Fluid Dyn. Res. 23, 189-234.

Goldshtik, M. A., Hussain, F. \& Shtern, V. N. 1991 Symmetry breaking in vortex-source and Jeffery-Hamel flows. J. Fluid Mech. 232, 521-566.

Govindarajan, R. \& Narasimha, R. 1995 Stability of spatially developing boundary layers in pressure gradients. J. Fluid Mech. 300, 117-147.

Hall, M. G. 1972 Vortex breakdown. Annu. Rev. Fluid Mech. 4, 125-218.

Kambe, T. 1969 The stability of an axisymmetric jet with a parabolic profile. J. Phys. Soc. Japan 26, 566-575.

Keller, J. J., EgLI, W. \& ExLey, W. 1985 Force- and loss-free transitions between flow states. Z. Agnew. Math. Phys. 36, 856-889.

Khorrami, M. R. \& Trivedi, P. 1994 The viscous stability analysis of Long's vortex. Phys. Fluids 6, 2623-2630. 
LaNDAU, L. D. 1944 On exact solution of the Navier-Stokes equations. Dokl. Akad. Nauk SSSR 43, 299-301.

Landau, L. D. \& Lifshitz, E. M. 1987 Fluid Dynamics, 2nd edn. Pergamon.

Lessen, M \& Singh, P. J. 1973 The stability of axisymmetric free shear layers. J. Fluid Mech. 60, 433-457.

LibBy, P. A. \& Fox, H. 1963 Some perturbation solutions in laminar boundary-layer theory. J. Fluid Mech. 268, 71-88.

LoNG, R. R. 1961 A vortex in an infinite viscous fluid. J. Fluid Mech. 11, 611-625.

McAlpine, A. \& Drazin, P. G. 1998 On the spatio-temporal development of small perturbations of Jeffery-Hamel flows. Fluid Dyn. Res. 22, 123-138.

Mollendorf, J. C. \& Gebhart, B. 1973 An experimental and numerical study of the viscous instability of a round laminar vertical jet with and without thermal buoyancy for symmetric and asymmetric disturbances. J. Fluid Mech. 61, 367-399.

Morris, P. J. 1976 The spatial viscous instability of axisymmetric jets. J. Fluid Mech. 77, 511-529.

Nathan, G. J., Hill, S. J. \& Luxton, R. E. 1998 An axisymmetric 'fluidic' nozzle to generate jet precession. J. Fluid Mech. 370, 347-380.

ReYnolds, A. J. 1962 Observations of a liquid-into-liquid jet. J. Fluid Mech. 14, 552-556.

Schlichting, H. 1933 Laminare Strahlausbreitung. Z. Angew. Math. Mech. 13, 260-263.

Shtern, V. \& Barrero, A. 1995 Bifurcation of swirl in liquid cones. J. Fluid Mech. 300, 169-205.

Shtern, V. \& Drazin, P. G. 2000 Instability of a free swirling jet driven by a half-line vortex. Proc. R. Soc. Lond. A 456, 1139-1161.

Shtern, V. \& Hussain, F. 1996 Hysteresis in swirling jets. J. Fluid Mech. 309, 1-44.

Shtern, V. \& Hussain, F. 1998 Instabilities of conical flows causing steady bifurcations. J. Fluid Mech. 366, 33-85 (referred to herein as SH98).

Shtern, V. \& Hussain, F. 2001 Generation of collimated jets by a point source of heat and gravity. J. Fluid Mech. 449, 39-59.

SQuire, H. B. 1952 Some viscous fluid flow problems. 1. Jet emerging from a hole in a plane wall. Phil. Mag. 43, 942-945.

Squire, H. B. 1956 Rotating fluids. In Surveys in Mechanics (ed. G. K. Batchelor \& R. M. Davies), pp. 139-61. Cambridge University Press.

Taм, K. K. 1996 Linear stability of the non-parallel Bickley jet. Can. Appl. Maths Q. 3, 99-110.

VIILU, A. 1962 An experimental determination of the minimum Reynolds number for instability in a free jet. J. Appl. Mech. 29, 506-508. 